\title{
Effects of the cosmological constant on cold dark matter clusters
}

\author{
M. Membrado ${ }^{1}$ and A. F. Pacheco ${ }^{2}$ \\ 1 Departamento de Física Teórica, Universidad de Zaragoza, 50009 Zaragoza, Spain \\ e-mail: membrado@unizar.es \\ 2 BIFI, Instituto de Biofísica y Física de Sistemas Complejos, Universidad de Zaragoza, 50009 Zaragoza, Spain \\ e-mail: amalio@unizar.es
}

Received 28 February 2014 / Accepted 2 May 2014

\begin{abstract}
Context. Cold dark matter inhomogeneities are considered in a homogeneous background of matter, radiation, and the cosmological constant in a flat universe.

Aims. We investigate the influence of the cosmological constant on the non-linear collapse of cold dark matter clusters.

Methods. For simplicity, a spherical infall model has been used to describe the collapse of non-relativistic mass shells; besides, an average distribution of density around a cluster of galaxies has been taken. Boundary conditions are imposed by the solution of the linearized equation for the growth of matter perturbations and by the cold dark matter power spectrum.

Results. For an average cluster, the radii of shells and masses enclosed by them have been obtained at their zero proper acceleration (ZA) redshifts, at their turn-around (TA) redshifts and at their virialization (VIR) redshifts. According to our results at present, the shell that reaches its turn-around point shows $\left[r^{\mathrm{TA}}\right]_{0}=6.85 \mathrm{Mpc}$ and $\left[\mathcal{M}^{\mathrm{TA}}\right]_{0}=6.76 \times 10^{14} \mathcal{M}_{\odot}$. The virializing shell fulfills $\left[r^{\mathrm{TA}}\right]_{0}=4.57\left[r^{\mathrm{VIR}}\right]_{0}$ and $\left[\mathcal{M}^{\mathrm{TA}}\right]_{0}=1.95\left[\mathcal{M}^{\mathrm{VIR}}\right]_{0}$. These results differ appreciably from those derived from a model with cosmological constant equal to zero in a flat universe: $\left[r^{\mathrm{TA}}(\Lambda=0)\right]_{0}=6.62\left[r^{\mathrm{VIR}}(\Lambda=0)\right]_{0}$ and $\left[\mathcal{M}^{\mathrm{TA}}(\Lambda=0)\right]_{0}=5.26\left[\mathcal{M}^{\mathrm{VIR}}(\Lambda=0)\right]_{0}$; this discrepancy could be considered as a new independent proof of the existence of dark energy. The shell with zero proper acceleration presents $\left[r^{\mathrm{ZA}}\right]_{0}=1.59\left[r^{\mathrm{TA}}\right]_{0}$ and $\left[\mathcal{M}^{\mathrm{ZA}}\right]_{0}=1.63\left[\mathcal{M}^{\mathrm{TA}}\right]_{0}$. We have found that there is a limit to the mass of the average cluster, which is able to virialize; its value is $\left\{\mathcal{M}^{\mathrm{VIR}}\right\}_{\mathrm{MAX}}=8.1 \times 10^{14} M_{\odot}$. As expected, we found that shells present null proper acceleration at redshift values that are smaller than 0.755 .

Conclusions. We have noticed that the cosmological constant imposes an upper limit for the mass enclosed by shells, which are able to reach zero proper velocity. Hence, this mass is the maximum mass of the virialized core, $\left\{\mathcal{M}^{\mathrm{VIR}}\right\}_{\mathrm{MAX}}$. For the average cluster addressed in this work, the value is 2.34 times the mass of the virialized core at present. Shells enclosing masses $\mathcal{M}>\left\{\mathcal{M}^{\mathrm{VIR}}\right\}_{\mathrm{MAX}}$ achieve zero proper acceleration and speed up, moving away from the virialized core, and never reach a turn-around point. Shells with $\mathcal{M} \gg\left\{\mathcal{M}^{\mathrm{VIR}}\right\}_{\text {MAX }}$ show zero proper aceleration at redshifts close to that at which the universe background acceleration is null. Finally, we have found that the relation between shell proper velocities and their radii can be adjusted by a straight line at $z=0$ and from approximately 20 up to $40 \mathrm{Mpc}$; however, this line does not intercept the origin as velocities due to the Hubble flux do.
\end{abstract}

Key words. dark matter - dark energy - galaxies: clusters: general

\section{Introduction}

It is accepted that more mass than visible matter is required to explain the observed structure in the universe. The first argument in favor of its existence was that non-baryonic dark matter was necessary to describe the rotation curves observed in galaxies (see, for example, Caldwell 1995), but the main argument comes from the primordial or Big Bang nucleosynthesis. In addition, dark matter models are consistent with other cosmological observations: the angular power spectrum of the cosmic microwave background, the large structure of the universe, and lensing clusters (see, for example: Bond 1988; Strukov et al. 1987; Trimble 1987; Peacock 1999).

At the end of the last century, it was realized that another new component of the universe had to be considered. If our universe, which seems to be flat (Efstathiou et al. 1998), contained just radiation and matter, it would be too young to be consistent with the ages of the oldest stars (Chaboyer et al. 1995).
This new component was named "dark energy", and its pressure (from a hydrodynamical point of view) must be a negative function of its density. This new component not only solves the flatness dilemma but also other cosmological problems such as the strong evidence that the expansion of the Universe is speeding up (see, for example, Perlmutter et al. 1998, 1999; Schmidt et al. 1998).

It is well known from observations that the expansion of the universe is present even on scales of galactic clusters but somewhat diminished. The first confirmations came from studies of the motion of our Galaxy (a revision can be found, for example, in Huchra 1988). These studies indicated that the Local Group shows a proper velocity with respect to the core of the Virgo cluster of about $1000 \mathrm{~km} \mathrm{~s}^{-1}$ (the velocity of the Hubble flow is about $1200 \mathrm{~km} \mathrm{~s}^{-1}$ ). It should be said in this respect that the greatest virialized structures in the universe are the cores of galaxy clusters. Outside the virialized region, the matter is collapsing toward the core. The evolution of the density and infall 
velocity profiles around clusters of galaxies has been extensively discussed in the literature (e.g., Gunn \& Gott 1972; Peebles 1984; Weinberg 1987; Lahav et al. 1991), and observational and theoretical advances have been obtained (e.g., Yahil et al. 1980; Davis \& Huchra 1982; Lilje \& Lahav 1991; Fouqué et al. 2001; Karachentsev \& Nasovona 2010). The Virgo infall region is one of the most extensively studied (see, for example: Silk 1974, 1977; Peebles 1976; Hoffman \& Salpeter 1982; Sandage 1986; Teerikorpi et al. 1992; Ekholm et al. 1999, 2000).

The aim of this work is to study how the cosmological constant affects the collapse of cold dark matter (CDM) clusters. In the spherical infall model (see, for example, Gunn \& Gott 1972; Peebles 1984; Weinberg 1987), mass spherical shells, which initially followed the expansion of the universe, begin to slow down due to the gravitational attraction of the mass they enclose. Some shells continue to slow down, reaching proper velocities equal to zero. When this happens, their radii are at a maximum. After reaching these turn-around radii, their proper velocities become negative. These shells end up virializing.

However, this should not occur for all shells. Each cluster should have a limiting shell with a radius enclosing the maximum mass, which allows a turn-around point to be reached. This is a consequence of the repulsive effect of the dark energy. If the mass enclosed by a shell is greater than this mass limit, there will be a redshift, at which the proper acceleration is null. At the zero proper acceleration radius, the attractive gravitational effect of the mass enclosed by the shell is balanced by the repulsive effect of the dark energy. At smaller redshifts, the proper velocity will remain positive, and the shell will keep expanding without reaching a turn-around radius.

In Sect. 2, we look at the limit imposed by the cosmological constant on the size of a bound non-relativistic cluster. In Sect. 3, the zero acceleration redshift for background non-relativistic matter is described. Section 4 deals with the collapse of CDM clusters within a background of matter, radiation and the cosmological constant. A discussion of the Hubble-Sandage paradox appears in Sect. 5. Finally, our conclusions are presented in Sect. 6.

\section{The effect of the cosmological constant on bound non-relativistic systems}

For a point mass, $\mathrm{m}$, there is a critical distance, $\mathrm{d}_{\mathrm{c}}$, where the attractive gravitational effect of the particle is balanced by the repulsion exerted by the cosmological constant, $\Lambda$. This critical distance is (see, for example, Membrado \& Pacheco 2012)

$d_{\mathrm{c}}=\left(\frac{3 G m}{\Lambda c^{2}}\right)^{1 / 3}$

The same result is derived for the critical radius, $R_{\mathrm{c}}$, of a bound spherically symmetric cluster containing a mass $\mathcal{M}$ of nonrelativistic matter, where attractive and repulsive strengths are compensated. In this case, $R_{\mathrm{c}}$ is obtained by imposing the condition $\mathrm{d} \phi /\left.\mathrm{d} r\right|_{R_{\mathrm{c}}}=0$, where $\phi$ is the gravitational potential and $r$ the radial distance. The value of $\mathrm{d} \phi / \mathrm{d} r$ can be obtained from the radial integration of the weak field approximation of the Einstein equations for ideal fluids (see, for example, Peebles 1980, Sect. 6, p. 39):

$\frac{1}{r^{2}} \frac{\mathrm{d}}{\mathrm{d} r}\left(r^{2} \frac{\mathrm{d} \phi}{\mathrm{d} r}\right)=\frac{4 \pi G}{c^{2}}(\rho+3 p)$, where $\rho$ and $p$ are the total energy density and pressure, respectively, of the fluids. By treating dark energy from a hydrodynamical point of view and by assuming a the cosmological constant model,

$\rho=\rho_{\mathrm{m}}+\bar{\rho}_{\Lambda}$

$p=\bar{p}_{\Lambda}$.

In Eqs. (3) and (4), $\rho_{\mathrm{m}}$ is the energy density of the non-relativistic matter, $\bar{\rho}_{\Lambda}$, the energy density, and $\bar{p}_{\Lambda}$, the pressure of dark energy. The values of $\bar{\rho}_{\Lambda}$ and $\bar{p}_{\Lambda}$ can be deduced by imposing that the energy-momentum tensor for $\Lambda$ is $\left[T^{\mu \nu}\right]_{\Lambda}=\frac{\Lambda c^{2}}{8 \pi G} g^{\mu \nu}$ (so, $\left[T_{; v}^{\mu v}\right]_{\Lambda}=0$ ), for any metric $g_{\mu v}$. Thus, assuming an ideal fluid (i.e., $\left[T^{\mu v}\right]_{\Lambda}=-\bar{p}_{\Lambda} g^{\mu \nu}+\left(\bar{\rho}_{\Lambda}+\bar{p}_{\Lambda}\right) U^{\mu} U^{v} ; U^{\mu}=\mathrm{d} x^{\mu} / \mathrm{d} s$ being the four-velocity tensor; $x^{\mu}$, the four-coordinate tensor; and $\mathrm{d} s$, the line element which fulfills $\left.(\mathrm{d} s)^{2}=g_{\mu \nu} \mathrm{d} x^{\mu} \mathrm{d} x^{\nu}\right)$,

$\bar{\rho}_{\Lambda}=-\bar{p}_{\Lambda}=\frac{c^{4}}{8 \pi G} \Lambda$.

Hence

$\left.\frac{\mathrm{d} \phi}{\mathrm{d} r}\right|_{R_{\mathrm{c}}}=0=\frac{G \mathcal{M}}{R_{\mathrm{c}}^{2}}-\frac{c^{2} \Lambda R_{\mathrm{c}}}{3}$

and, therefore,

$R_{\mathrm{c}}=\left(\frac{3 G \mathcal{M}}{\Lambda c^{2}}\right)^{1 / 3}$.

In a bound spherically symmetric cluster composed of nonrelativistic matter, the gradient of matter pressure balances the gravitational strength per volume unit (i.e. $\mathrm{d} p_{\mathrm{m}} / \mathrm{d} r=$ $\left.-\rho_{\mathrm{m}} \mathrm{d} \phi / \mathrm{d} r\right)$. This happens only if the cluster radius, $R$, is smaller than the critical radius, $R_{\mathrm{c}}$. If the cluster extends beyond $R_{\mathrm{c}}$ (where $G M\left(R_{\mathrm{c}}\right) / R_{\mathrm{c}}^{2}-c^{2} \Lambda R_{\mathrm{c}} / 3=0$ is fulfilled), then $\mathrm{d} \phi / \mathrm{d} r<0$ at $r>R_{\mathrm{c}}$; this is due to the repulsive effect of the cosmological constant. Therefore, the decreasing behavior of the pressure of the matter is not able to compensate for the gravitational effect, and the cluster expands according to the motion equation $\rho_{\mathrm{m}} \ddot{r}=-\mathrm{d} p_{\mathrm{m}} / \mathrm{d} r-\rho_{\mathrm{m}} \mathrm{d} \phi / \mathrm{d} r$. Hence, the maximum radius of a bound cluster of mass $\mathcal{M}$ is $R_{\mathrm{c}}$.

The value of $R_{\mathrm{c}}$, given by Eq. (7), coincides with that derived by Chernin et al. (see, for example: Chernin et al. 2000; Chernin 2001; Dolgachev et al. 2003, 2004) for the radius at which a dynamic spherically symmetric mass shell enclosing a mass, $\mathcal{M}$, shows null acceleration.

In previous papers (Membrado \& Pacheco 2012, 2013), we applied this upper limit to spheres of self-gravitating fermions, or bosons, in the ground state, which are quantum systems. These spheres simulated clusters of dark matter. As the equation of state of these systems is known, these bounds allowed to infer limiting values for the mass of the constituent elementary particle.

From Eq. (7), a critical mean energy density can also be defined,

$\rho_{\mathrm{c}}=\frac{3 \mathcal{M} c^{2}}{4 \pi R_{\mathrm{c}}^{3}}=\frac{c^{4} \Lambda}{4 \pi G}$.

Hence, the mean energy density of a bound non-relativistic system must be greater than this critical density.

Equation (8) can be expressed as a function of $\Omega_{\Lambda}=\bar{\rho}_{\Lambda} / \bar{\rho}_{0}$ and the Hubble function at present, $H_{0}=\left(8 \pi G \bar{\rho}_{0} / 3 c^{2}\right)^{1 / 2}$. In these equations, $\bar{\rho}_{0}$ is the background total energy density of 
the universe at present (hereafter, subindex 0 represents magnitudes at present; magnitudes with a bar represent background magnitudes). Thus,

$\rho_{\mathrm{c}}=1.243 \times 10^{-8}\left(\frac{\Omega_{\Lambda}}{0.73}\right)\left(\frac{H_{0}}{71 \mathrm{~km} \mathrm{~s}^{-1} \mathrm{Mpc}}\right)^{2} \mathrm{erg} \mathrm{cm}^{-3}$.

Values for $\Omega_{\Lambda}$ and $H_{0}$ can be taken from Spergel et al. (2003).

This critical density, $\rho_{\mathrm{c}}$, could be compared with the energy density of the expanding background non-relativistic matter of the universe at present,

$\bar{\rho}_{\mathrm{m} 0}=2.298 \times 10^{-9}\left(\frac{\Omega_{\mathrm{m} 0}}{0.27}\right)\left(\frac{H_{0}}{71 \mathrm{~km} \mathrm{~s}^{-1} \mathrm{Mpc}^{-1}}\right)^{2} \mathrm{erg} \mathrm{cm}^{3}$,

where $\Omega_{\mathrm{m} 0}=\bar{\rho}_{\mathrm{m} 0} / \bar{\rho}_{0}$ (a value for $\Omega_{\mathrm{m} 0}$ can also be taken from Spergel et al. 2003). Therefore, $\bar{\rho}_{\mathrm{m} 0}<\rho_{\mathrm{c}}$, and hence, at present, bound structures could not be built from perturbations of background non-relativistic matter.

\section{Zero acceleration in background non-relativistic matter}

For a homogeneous and isotropic background in a flat universe (Efstathiou et al. 1998), the line element, $\mathrm{d} s$, is given by the Friedmann-Robertson-Walker metric (see, for example, Raychaudhuri et al. 1992, Sect. 16.1, p. 212)

$(\mathrm{d} s)^{2}=(c \mathrm{~d} t)^{2}-a^{2}(\mathrm{~d} \boldsymbol{x})^{2}$.

In Eq. (11), $\boldsymbol{x}$ is the expanding coordinate, comoving in the background model; $a$ is the expansion parameter of the universe, and $t$ is the proper world time. Therefore, for such a background, any proper distance changes with time in proportion to the expanding parameter $a$, which is a universal function of the proper world time (see, for example, Padmanabhan 1995, Sect. 2.3, p. 54).

As a consequence, the radius of a sphere, expanding with the universe, evolves as $R(a)=\left(a / a_{0}\right) R_{0}$, where $a_{0}$ and $R_{0}$ are the expanding parameter and the sphere radius at present $\left(x=R_{0} / a_{0}\right.$ is the comoving radius; in other words, the expanding radius comoving in the background model). As the energy density of the background non-relativistic matter fulfills $\bar{\rho}_{\mathrm{m}}=\bar{\rho}_{\mathrm{m} 0}\left(a_{0} / a\right)^{3}$ (see, for example, Padmanabhan 1995, Sect. 2.4, p. 61), the matter mass, $\mathcal{M}$, inside the expanding sphere of radius $R(a)$ is constant.

According to the previous section, as the sphere of mass $\mathcal{M}$ expands, it could lead to bound structures while its radius, $R$, are smaller than the critical radius $R_{\mathrm{c}}$, given by Eq. (7). Using $\mathcal{M}=$ $\left(4 \pi / 3 c^{2}\right) \bar{\rho}_{\mathrm{m} 0} R_{0}^{3}$ and $R_{\mathrm{c}}=\left(a_{\mathrm{c}} / a_{0}\right) R_{0}=R_{0} /\left(1+z_{\mathrm{c}}\right)$, Eq. (7) tells us that any mass, $\mathcal{M}$, of background non-relativistic matter, reaches its critical radius, $R_{\mathrm{c}}$, at redshift $z_{\mathrm{c}}$ fulfilling

$1+z_{\mathrm{c}}=1.755\left(\frac{\Omega_{\Lambda}}{0.73}\right)^{1 / 3}\left(\frac{\Omega_{\mathrm{m} 0}}{0.27}\right)^{-1 / 3}$.

Therefore, bound structures cannot be created from perturbations of background non-relativistic matter arising at redshift $z<$ $z_{\mathrm{c}}$. Obviously, at this redshift, $\bar{\rho}_{\mathrm{m}}\left(a_{\mathrm{c}}\right)=\rho_{\mathrm{c}}$.

Let us now have a look at the Einstein equations for the evolution of the universe. Assuming the metric given by Eq. (11), these equations read as follows (see, for example, Padmanabhan 1995, Sect. 2.4, p. 60):

$\left(\frac{\dot{a}}{a}\right)^{2}=\frac{8 \pi G}{3 c^{2}} \bar{\rho}=H^{2}$

$\left(\frac{\ddot{a}}{a}\right)=-\frac{4 \pi G}{3 c^{2}}(\bar{\rho}+3 \bar{p})$.
In Eqs. (13) and (14), a dot represent $\mathrm{d} / \mathrm{d} t$ and $H$ is the Hubble function. Hence, if we consider a background composed of nonrelativistic matter and the cosmological constant, the universe shows zero acceleration (i.e., $\ddot{a}=0$ ) at redshift, $z_{U}^{\mathrm{ZA}}$, fulfilling

$1+z_{U}^{\mathrm{ZA}}=\left(\frac{2 \bar{\rho}_{\Lambda}}{\bar{\rho}_{\mathrm{m} 0}}\right)^{1 / 3}=1.755\left(\frac{\Omega_{\Lambda}}{0.73}\right)^{1 / 3}\left(\frac{\Omega_{\mathrm{m} 0}}{0.27}\right)^{-1 / 3}$.

This redshift given by Eq. (15) is, as expected, the same as that given by Eq. (12); i.e., $z_{\mathrm{c}}=z_{U}^{\mathrm{ZA}}$. For $z<z_{U}^{\mathrm{ZA}}$, the universe speeds up and bound structures can no longer be built from perturbations of background non-relativistic matter.

\section{The case of collapsing cold dark matter}

In this section, we focus our attention on the collapse of CDM. For simplicity, the collapse is treated with a spherical infall model (see, for example, Peebles 1980, Sect. 19). Thus, we study the collapse of a CDM spherical shell (assuming that no other mass shell crosses it) by integrating its motion equation in a background of matter, radiation and the cosmological constant. The integration is started at redshift $z_{\mathrm{I}}=1000$. At this redshift, the recombination has just occurred, and the growth of perturbations is fully given by the linear theory. Besides, the decay modes are negligible, and the velocity field is given by the linear theory, irrespective of initial velocities.

The first subsection is devoted to showing the equation of motion of a spherical shell enclosing a mass composed of nonrelativistic matter. The resolution of this equation allows us to follow the non-linear collapse of each mass shell. However, to solve it, it is necessary to know the radius and velocity of each shell at $z_{\mathrm{I}}$. In Sects. 4.2 and 4.3 we deal with the Newtonian theory of perturbations to fix the value of both magnitudes. Finally, Sect. 4.4 shows the numerical results.

\subsection{The equation of motion of a collapsing spherical shell}

Let us consider a spherical shell $i$ enclosing a mass $\mathcal{M}_{i}$, which at the expanding parameter $a_{\mathrm{I}}=a_{0} /\left(1+z_{\mathrm{I}}\right)$ has a radius $r_{i}\left(a_{\mathrm{I}}\right)$. At a proper distance $r^{\prime}\left(a_{\mathrm{I}}\right) \leq r_{i}\left(a_{\mathrm{I}}\right)$, which is at an expanding distance $x^{\prime}=r^{\prime}\left(a_{\mathrm{I}}\right) / a_{\mathrm{I}}$ from the center, the energy density of non-relativistic matter can be expressed as

$\rho_{\mathrm{m}}\left(x^{\prime}, a_{\mathrm{I}}\right)=\bar{\rho}_{\mathrm{m}}\left(a_{\mathrm{I}}\right)\left[1+\delta\left(x^{\prime}, a_{\mathrm{I}}\right)\right]$.

In Eq. (16), $\delta\left(x^{\prime}, a_{\mathrm{I}}\right)$ is the density contrast at the expanding radius $x^{\prime}$ and at expanding parameter $a_{\mathrm{I}}$, given by the linear theory of perturbations. Hence, the mass, $\mathcal{M}_{i}$, which is constant in the collapse, is

$\mathcal{M}_{i}=\frac{4 \pi G}{3 c^{2}} r_{i}^{3}\left(a_{\mathrm{I}}\right) \bar{\rho}_{\mathrm{m}}\left(a_{\mathrm{I}}\right)\left[1+\Delta_{i}\left(a_{\mathrm{I}}\right)\right]$.

In Eq. (17),

$\Delta_{i}\left(a_{\mathrm{I}}\right)=\frac{3}{\left[x_{i}\left(a_{\mathrm{I}}\right)\right]^{3}} \int_{0}^{x_{i}\left(a_{\mathrm{I}}\right)} \delta\left(x^{\prime}, a_{\mathrm{I}}\right) x^{\prime 2} \mathrm{~d} x^{\prime}$

is the average density contrast at $a_{\mathrm{I}}$, evaluated up to the expanding radius of the shell, $x_{i}\left(a_{\mathrm{I}}\right)=r_{i}\left(a_{\mathrm{I}}\right) / a_{\mathrm{I}}$.

For $a>a_{\mathrm{I}}$, the evolution of the radius of the shell $i, r_{i}(a)$, is obtained by solving the equation of motion

$\ddot{r}_{i}=-\frac{\mathrm{d} \phi}{\mathrm{d} r_{i}}$ 
where $\ddot{r}_{i}$ is the proper acceleration of the shell $i$ and $\phi$ is the gravitational potential. From the weak field approximation of the Einstein equations (see, for example, Peebles 1980, Sect. 6, p. 39), $\phi$ fulfills $\nabla^{2} \phi=\frac{4 \pi G}{c^{2}}\left[\left(\rho_{\mathrm{m}}+\bar{\rho}_{\gamma}+\bar{\rho}_{\Lambda}\right)+3\left(\bar{p}_{\gamma}+\bar{p}_{\Lambda}\right)\right]$, where a background of non-relativistic matter $\left(\bar{p}_{\mathrm{m}} \ll \bar{\rho}_{\mathrm{m}}\right)$, radiation $\left(\bar{p}_{\gamma}=\bar{\rho}_{\gamma} / 3\right)$ and the cosmological constant $\left(\bar{p}_{\Lambda}=-\bar{\rho}_{\Lambda}\right)$ has been assumed; hence, Eq. (19) reads as

$\ddot{r}_{i}=-\frac{G}{r_{i}^{2}} \mathcal{M}_{i}-\frac{4 \pi G}{3 c^{2}}\left[\left(\bar{\rho}_{\gamma}+3 \bar{p}_{\gamma}\right)+\left(\bar{\rho}_{\Lambda}+3 \bar{p}_{\Lambda}\right)\right] r_{i}$.

For each shell $i$ having a mass $\mathcal{M}_{i}$, Eq. (20) must be solved with the boundary conditions at $a_{\mathrm{I}}$, which is, from the values of the shell radius, $r_{i}\left(a_{\mathrm{I}}\right)$, and the proper velocity of the shell, $\dot{r}_{i}\left(a_{\mathrm{I}}\right)$. From Eq. (17),

$r_{i}\left(a_{\mathrm{I}}\right)=\left[\frac{3 c^{2} \mathcal{M}_{i}}{4 \pi G \bar{\rho}_{\mathrm{m}}\left(a_{\mathrm{I}}\right)\left[1+\Delta_{i}\left(a_{\mathrm{I}}\right)\right]}\right]^{1 / 3}$.

With respect to the proper velocity of the mass shell at $a_{\mathrm{I}}$, it can be expressed as

$\dot{r}_{i}\left(a_{\mathrm{I}}\right)=H\left(a_{\mathrm{I}}\right) r_{i}\left(a_{\mathrm{I}}\right)+v_{i}\left(a_{\mathrm{I}}\right)$.

In Eq. (22), the first term on the right is the velocity due to the Hubble flow; and the second term, $v_{i}\left(a_{\mathrm{I}}\right)$, is the peculiar velocity of the shell at $a_{\mathrm{I}}$, given by

$v_{i}\left(a_{\mathrm{I}}\right)=a_{\mathrm{I}} \dot{x}_{i}\left(a_{\mathrm{I}}\right)$.

We denote by $\delta_{\mathrm{g}}$ the growing solution of the linearized Newtonian equation for the growth of matter perturbations (see next subsection). It can be shown that for this $\delta_{\mathrm{g}}$, the peculiar velocity of a shell $i$ at the expanding parameter $a_{i}$, is given by (from, for example, Eqs. (14.6) and (14.2) from Peebles 1980, Sect. 14)

$v_{i}\left(a_{\mathrm{I}}\right)=-\frac{1}{3} f_{\mathrm{g}}\left(a_{\mathrm{I}}\right) H\left(a_{\mathrm{I}}\right) \Delta_{i}\left(a_{\mathrm{I}}\right) r_{i}\left(a_{\mathrm{I}}\right)$

where

$f_{\mathrm{g}}\left(a_{\mathrm{I}}\right)=\left.\frac{a_{\mathrm{I}}}{\delta_{\mathrm{g}}\left(a_{\mathrm{I}}\right)} \frac{\mathrm{d} \delta_{\mathrm{g}}}{\mathrm{d} a}\right|_{a_{\mathrm{I}}}$.

Let us come back to Eq. (20). Defining the radius of the shell for any time as

$r_{i}(a)=A_{i}(a) r_{i}\left(a_{\mathrm{I}}\right)=A_{i}(a) a_{\mathrm{I}} x_{i}\left(a_{\mathrm{I}}\right)$,

and taking derivatives with respect to $a$, Eq. (20) reads as

$$
\begin{aligned}
a^{2} \frac{\bar{\rho}}{\bar{\rho}_{0}} A_{i}^{\prime \prime}= & -a A_{i}^{\prime}\left[\frac{\bar{\rho}}{\bar{\rho}_{0}}+\frac{1}{2} a \frac{\bar{\rho}^{\prime}}{\bar{\rho}_{0}}\right]-\frac{1}{2 A_{i}^{2}} \frac{\bar{\rho}_{\mathrm{m}}\left(a_{\mathrm{I}}\right)}{\bar{\rho}_{0}}\left[1+\Delta_{i}\left(a_{\mathrm{I}}\right)\right] \\
& -\frac{A_{i}}{2}\left[\frac{\bar{\rho}_{\gamma}+\bar{\rho}_{\Lambda}}{\bar{\rho}_{0}}+3 \frac{\bar{p}_{\gamma}+\bar{p}_{\Lambda}}{\bar{\rho}_{0}}\right] .
\end{aligned}
$$

This Eq. (27) must be solved with the boundary conditions

$A_{i}\left(a_{\mathrm{I}}\right)=1$,

$A_{i}^{\prime}\left(a_{\mathrm{I}}\right)=\frac{1}{a_{\mathrm{I}}}\left[1-\frac{1}{3} f_{\mathrm{g}}\left(a_{\mathrm{I}}\right) \Delta_{i}\left(a_{\mathrm{I}}\right)\right]$

(see, Eqs. (21)-(25)).

\subsection{The contrast density $\delta\left(x, a_{1}\right)$}

It is assumed that, in the past, there were small deviations from the homogeneous background of the Universe, which grew due to gravitational instabilities. While such inhomogeneities are small, their growth can be studied by the linear perturbation theory. When linear theory fails, the evolution must be studied by non-linear methods, such as that proposed in the previous subsection.

As inhomogeneities lead to small perturbations of the energy-momentum tensor and of the metric, the Einstein equations can be linearized. The result is a second-order equation. Then, its solution can be expanded in terms of some mode functions. For a flat universe, the mode functions are plane waves. Hence, by using a Fourier transformation of the variables, the evolution of each mode, characterized by a wave vector, $\boldsymbol{k}$, can be determined separately.

When the proper length of a mode at some expanding parameter, $a$, is greater than the Hubble length at that $a$, the general relativistic perturbation theory is applied; that is, for a mode $\lambda=(2 \pi) / k$, such a theory must be applied if $a<a_{\lambda}$, where $a_{\lambda}$ fulfills

$\lambda a_{\lambda}=\frac{c}{H\left(a_{\lambda}\right)}$.

Therefore, the evolution of that mode can be known from some initial expanding parameter up to $a_{\lambda}$. For $a>a_{\lambda}$, the evolution of the perturbation can be studied from the Newtonian theory.

In this subsection, we deal with non-relativistic matter, which is slightly perturbed, from the cosmological background in a region where the Newtonian approximation can be applied.

Defining the density contrast, $\delta(\boldsymbol{x}, a)$, by $\rho_{\mathrm{m}}(\boldsymbol{x}, a)=$ $\bar{\rho}_{\mathrm{m}}(a)[1+\delta(\boldsymbol{x}, a)]$ (see Eq. (16)), the linearized equation for the growth of matter perturbations is then given by (see, for example, Peebles 1980, Sect. 10, p. 49)

$\frac{\bar{\rho}}{\bar{\rho}_{0}} a^{2} \frac{\partial^{2} \delta}{\partial a^{2}}+\left[3 \frac{\bar{\rho}}{\bar{\rho}_{0}}+\frac{1}{2} \frac{a}{\bar{\rho}_{0}} \frac{\mathrm{d} \bar{\rho}}{\mathrm{d} a}\right] a \frac{\partial \delta}{\partial a}=\frac{3}{2} \Omega_{\mathrm{m} 0}\left(\frac{a}{a_{0}}\right)^{-3} \delta$.

The only differentiations that appear in Eq. (31), are those with respect to the expansion parameter, $a$. So we have denoted the density contrast just by $\delta$; that is, $\delta \equiv \delta(a, x)$. In the same equation, $\bar{\rho}$ is the total energy density of the background, which we are assuming to be (see, for example, Padmanabhan 1995, Sect. 2.4, p. 61)

$\bar{\rho}=\bar{\rho}_{\mathrm{m} 0}\left(\frac{a_{0}}{a}\right)^{3}+\bar{\rho}_{\gamma 0}\left(\frac{a_{0}}{a}\right)^{4}+\bar{\rho}_{\Lambda}$.

In Eq. (32): $\bar{\rho}_{\mathrm{m} 0}=\bar{\rho}_{0} \Omega_{\mathrm{m} 0}=\left(3 c^{2} / 8 \pi G\right) \Omega_{\mathrm{m} 0} H_{0}^{2}$ (see Eq. (13)); $\bar{\rho}_{\gamma 0}=\left(\pi^{2} / 15\right)\left[\left(K_{\mathrm{B}} T_{\gamma 0}\right)^{4} /(\hbar c)^{3}\right]$ (see, for example, Padmanabhan 1995, Sect. 3.2, p. 86), where $K_{\mathrm{B}}$ is the Boltzmann constant and $T_{\gamma 0}=2.75 \mathrm{~K}$ is the temperature of the background radiation at present (see, for example, Padmanabhan 1995, Sect. 1.9, p. 35, and references there in); and $\bar{\rho}_{\Lambda}=\bar{\rho}_{0} \Omega_{\Lambda}=\left(3 c^{2} / 8 \pi G\right) \Omega_{\Lambda} H_{0}^{2}$. Thus,

$\bar{\rho}_{\mathrm{m} 0}=2.298 \times 10^{-9}\left(\frac{\Omega_{\mathrm{m} 0}}{0.27}\right)\left(\frac{H_{0}}{71 \mathrm{~km} \mathrm{~s}^{-1} \mathrm{Mpc}^{-1}}\right)^{2} \mathrm{erg} \mathrm{cm}^{-3}$,

$\bar{\rho}_{\gamma 0}=4.338 \times 10^{-13} \mathrm{erg} \mathrm{cm}^{3}$,

and

$\bar{\rho}_{\Lambda}=6.213 \times 10^{-9}\left(\frac{\Omega_{\Lambda}}{0.73}\right)\left(\frac{H_{0}}{71 \mathrm{~km} \mathrm{~s}^{-1} \mathrm{Mpc}^{-1}}\right)^{2} \mathrm{erg} \mathrm{cm}^{-3}$. 
It should be said that the radiation term in Eq. (32) is only valid for $a>a_{\mathrm{e}^{+} \mathrm{e}^{-}}$, where $a_{\mathrm{e}^{+} \mathrm{e}^{-}} \approx a_{0}\left(K_{\mathrm{B}} T_{\gamma_{0}} / m_{\mathrm{e}^{-}} c^{2}\right)$ is the expansion parameter at which $\mathrm{e}^{+} \mathrm{e}^{-}$annihilation takes place; which is, for

$\frac{a}{a_{0}}>\frac{a_{\mathrm{e}^{+} \mathrm{e}^{-}}}{a_{0}} \approx 4.639 \times 10^{-10}$.

Equation (31) is a second order differential equation having two linearly independent solutions: a growing solution, $\delta_{\mathrm{g}}$, and a decaying solution, $\delta_{\mathrm{d}}$. A general solution for a mode $\boldsymbol{k}$, is a linear superposition of both; that is, $\delta_{k}(a)=A_{k} \delta_{\mathrm{g}}(a)+B_{k} \delta_{\mathrm{d}}(a)$. The constants $A_{k}$ and $B_{k}$ are fixed by matching at $a_{\lambda}$, the Newtonian solution to the growing solution of the general relativistic perturbation equation, which was valid when the mode was bigger than the Hubble length (see, for example, Padmanabhan 1995, Sect. 4).

In this study, we are interested in the non-linear evolution of growing modes from $z_{\mathrm{I}}=1000$. At this redshift, the mode having a wavelength equal to the Hubble length is the mode labeled by $\lambda_{\max }$ with (see Eq. (30))

$\lambda_{\max } a_{0}=\left(\frac{a_{0}}{a_{I}}\right)\left(\frac{c}{H\left(a_{I}\right)}\right) \approx 2.356 \times 10^{2} \mathrm{Mpc}$

(Eqs. (13) and (32)-(35) are used, and $\Omega_{\mathrm{m} 0}=0.27$ and $\Omega_{\Lambda}=$ 0.73 have been assumed).

Thus, at $a_{\mathrm{I}}$, growing Newtonian modes $\lambda<\lambda_{\max }$ fulfill

$\delta_{k}\left(a_{\mathrm{I}}\right) \approx A_{k} \delta_{\mathrm{g}}\left(a_{\mathrm{I}}\right)$.

In this work, we assume that the density contrast, $\delta\left(\boldsymbol{x}, a_{\mathrm{I}}\right)$, can be expressed as

$\delta\left(\boldsymbol{x}, a_{\mathrm{I}}\right)=\frac{\delta_{\mathrm{g}}\left(a_{\mathrm{I}}\right)}{\delta_{\mathrm{g}}\left(a_{0}\right)} D(\boldsymbol{x})$,

where $D(\boldsymbol{x})$ is the density field given by

$D(\boldsymbol{x})=\frac{1}{(2 \pi)^{3}} \int \delta_{k} \mathrm{e}^{-\mathrm{i} \boldsymbol{k} \cdot \boldsymbol{x}} \mathrm{d}^{3} k$,

which considers all the $\lambda$ modes. The $\delta_{k}$ 's are, therefore, the Fourier components of the density field. Thus, the density field is completely determined by the power spectrum, $P_{k}=\left|\delta_{k}\right|^{2}$. In this study, instead of dealing with $D(\boldsymbol{x})$ given by Eq. (41), we work with the average density field $\langle D(x)\rangle$. Section 4.3 is devoted to this.

In this work, we are interested in the study of the non-linear spherical collapse of Newtonian mass shells from $a_{\mathrm{I}}$. Therefore, we only deal with shells that have sizes smaller than the Hubble length at $a_{\mathrm{I}}$; that is, shells whose radii at $a_{\mathrm{I}}, R\left(a_{\mathrm{I}}\right)$, fulfill

$R\left(a_{\mathrm{I}}\right)<1.178 \times 10^{2}\left(\frac{a_{\mathrm{I}}}{a_{0}}\right) \mathrm{Mpc}$,

which enclose masses

$\mathcal{M}=\frac{4 \pi \bar{\rho}_{\mathrm{m} 0}\left(a_{\mathrm{I}}\right)}{3 c^{2}} R\left(a_{\mathrm{I}}\right)^{3}<2.585 \times 10^{17} \mathcal{M}_{\odot}$.

The growing solution of Eq. (31), assuming Eq. (32), must be derived from an adequate boundary condition. This can be obtained from the solution at the epoch where radiation dominates the background energy densities, that is. at $a \ll a_{\text {eq }}$, where

$$
\frac{a_{\mathrm{eq}}}{a_{0}}=\frac{\bar{\rho}_{\gamma 0}}{\bar{\rho}_{\mathrm{m} 0}}=1.888 \times 10^{-4}\left(\frac{\Omega_{\mathrm{m} 0}}{0.27}\right)^{-1}\left(\frac{H_{0}}{71 \mathrm{~km} \mathrm{~s}^{-1} \mathrm{Mpc}^{-1}}\right)^{-2}
$$

It is easy to show that this asymptotic growing solution for $a_{\mathrm{e}^{+} \mathrm{e}^{-}}<a \ll a_{\mathrm{eq}}$ is given by

$\delta_{\mathrm{g}}(a) \propto 1+\frac{3}{2} \frac{a}{a_{\mathrm{eq}}}$.

Thus, we have found that the growing solution of Eq. (31) at the expanding parameter $a_{\mathrm{I}}$ fulfills

$$
\begin{aligned}
& \frac{\delta_{\mathrm{g}}\left(a_{\mathrm{I}}\right)}{\delta_{\mathrm{g}}\left(a_{0}\right)}=1.48 \times 10^{-3}, \\
& \left.\frac{a_{\mathrm{I}}}{\delta_{\mathrm{g}}\left(a_{\mathrm{I}}\right)} \frac{\mathrm{d} \delta_{\mathrm{g}}}{\mathrm{d} a}\right|_{a_{\mathrm{I}}}=8.88 \times 10^{-1} .
\end{aligned}
$$

\subsection{The density field $D(x)$}

To evaluate $\Delta_{i}\left(a_{\mathrm{I}}\right)$ from Eq. (18), we need to know the profile of the collapsing structure at $a_{\mathrm{I}}$. Such a profile is determined by Eq. (39). The quotient $\delta_{\mathrm{g}}\left(a_{\mathrm{I}}\right) / \delta_{\mathrm{g}}\left(a_{0}\right)$ was already calculated in the previous subsection (see (45)). In this subsection, we deal with the second function appearing in (39), the density field, $D(x)$.

In our study, the progenitors of clusters of galaxies are identified with certain peaks in the Gaussian postrecombination density field. The height of these peaks, $D(x=0)$, should be higher than a certain threshold.

Bardeen et al. (1986; hereafter BBKS) studied the statistics of the distribution of the density field around a peak in a linear Gaussian field. In this work, the average density profile of peaks proposed by Lilje \& Lahav (1991; see Eq. (3.1)), based on the work by BBKS, is assumed for $\langle D(x)\rangle$.

Thus, for a peak with a height

$D(0)=v \sigma_{0}$,

where

$\sigma_{0}^{2}=\frac{1}{2 \pi^{2}} \int_{0}^{\infty} P_{k} k^{2} \mathrm{~d} k$

is the rms fluctuations of the density field, the adopted expression for $\langle D(x)\rangle$ is

$$
\begin{aligned}
\langle D(x)\rangle= & \frac{1}{2 \pi^{2} \sigma_{0}} \int_{0}^{\infty} k^{2} P_{k}\left[\frac{v-\gamma^{2} v-\gamma \chi}{1-\gamma^{2}}+\frac{\chi R_{\star}^{2} k^{2}}{3 \gamma\left(1-\gamma^{2}\right)}\right] \\
& \times \frac{\sin k x}{k x} \mathrm{~d} k .
\end{aligned}
$$

In Eq. (49),

$$
R_{\star}=\sqrt{3} \frac{\sigma_{1}}{\sigma_{2}}, \quad \gamma=\frac{\sigma_{1}^{2}}{\sigma_{2} \sigma_{0}}
$$

with

$\sigma_{i}^{2}=\frac{1}{2 \pi^{2}} \int_{0}^{\infty} P_{k} k^{2+2 i} \mathrm{~d} k$

and

$\chi=\frac{3\left(1-\gamma^{2}\right)+\left(1.216-0.9 \gamma^{4}\right) \mathrm{e}^{-\gamma / 2(\gamma \nu / 2)^{2}}}{\left[3\left(1-\gamma^{2}+0.45+(\gamma \nu / 2)^{2}\right]^{1 / 2}+\gamma \nu / 2\right.}$.

The term $\langle D(x)\rangle$ in Eq. (49) is expressed as a superposition of modes, $\delta_{k}$, with different wavenumbers, $k=(2 \pi) / \lambda$, where $P_{k}=\left|\delta_{k}\right|^{2}$ is the power spectrum. As we are interested in the collapse of CDM structures, we must deal with the CDM power spectrum. It so happens that there is not a natural cutoff 
for high wavenumber in such a spectrum, so we have to make use of a filter. In this work, we use a Gaussian filter. Thus,

$P_{k}=P_{0} k T_{k}^{2} \exp \left[-\left(R_{\mathrm{f}} k\right)^{2}\right]$.

In Eq. (53), $k$ is proportional to the initial spectrum taken as the Zeldovich spectrum that arises in inflation, and, $T_{k}^{2}$ is the transfer function for linear perturbations at later times. For CDM (adiabatic fluctuations), $T_{k}^{2}$ is given by the following numerical fitting formulae of Bardeen et al. (1986; see Eq. (G3)):

$T_{k}^{2}=\frac{[\ln (1+2.34 q) /(2.34 q)]^{2}}{\left[1+3.89 q+(16.1 q)^{2}+(5.46 q)^{3}+(6.71 q)^{4}\right]^{1 / 2}}$,

where

$q=\left(\frac{k}{1 h \mathrm{Mpc}^{-1}}\right) \frac{1}{\Gamma}$

and

$h=\left(\frac{H_{0}}{100 \mathrm{~km} \mathrm{~s}^{-1} \mathrm{Mpc}^{-1}}\right)$.

The parameter $\Gamma$ was originally defined by Efstathiou et al. (1992) for $\Omega_{\mathrm{m} 0}=1$ and $\Omega_{B 0}=\bar{\rho}_{B 0} / \bar{\rho}_{0}=0.03$ (for baryons) and generalized for $\Omega_{\mathrm{m} 0} \neq 1$ by Sugiyama (1995); in this study, we use the expression proposed by Sugiyama, given by

$\Gamma=h \Omega_{\mathrm{m} 0} \exp \left[-\Omega_{B 0}\left(1+\sqrt{2 h} / \Omega_{\mathrm{m} 0}\right)\right]$.

From a combination of WMAP data with other finer scale CMB experiments (ACBAR and CBI), 2dFGRS measurements, and Lya forest data, it is found that (Spergel et al. 2003) $h=$ $0.71_{-0.03}^{+0.04}, \Omega_{\mathrm{m} 0}=0.27 \pm 0.04$, and $\Omega_{B 0}=0.044 \pm 0.004$.

With respect to the filtering scale, $R_{\mathrm{f}}$, we take

$R_{\mathrm{f}}=3 h^{-1} \mathrm{Mpc}$

this value was proposed by Lilje \& Lahav (1991) for clusters of galaxies. Thus, we obtain

$$
\begin{aligned}
R_{\star} & =4.26 h^{-1} \mathrm{Mpc}, \\
\gamma & =6.50 \times 10^{-1} .
\end{aligned}
$$

It can be seen that the values of $R_{\star}$ and $\gamma$, given by Eqs. (59) and (60) are a little different to those derived by Lilje \& Lahav (1991; see Table 1) for their CDM model. Such a discrepancy is due to the value of $\Gamma$ used: we use that given by Eq. (57), while they used $\Gamma=h$.

In Eq. (53), $P_{0}$ is the normalization factor which can be determined from $\sigma_{8}$ (the present rms density fluctuations in the sphere with a radius $R=8 h^{-1} \mathrm{Mpc}$; see, for example, Peebles 1980 , Sect. 26, p. 121), which is given by

$\sigma_{8}^{2}=\int_{0}^{\infty} k^{2} \frac{P_{0} k T_{k}^{2}}{2 \pi^{2}}\left[\frac{3(\sin k R-k R \cos k R)}{(k R)^{3}}\right]^{2} \mathrm{~d} k$.

The value of $\sigma_{8}$ can be derived by fitting the theoretically predicted number density of clusters versus temperature (cumulative temperature function) to the observed X-ray cluster abundance as a function of temperature. In this work, we use the fitting by Membrado \& Aguerri (2004); i.e., we assume $\sigma_{8}=$ $0.49 \Omega_{\mathrm{m} 0}^{-0.22 \Omega_{\mathrm{m} 0}-0.35}$, which for $\Omega_{\mathrm{m} 0}=0.27$, gives $\sigma_{8}=0.84$. Thus, we obtain

$P_{0}=4.05 \times 10^{6} h^{-4} \mathrm{Mpc}^{4}$.
Here, the threshold, $\nu_{\mathrm{T}} \sigma_{0}$, is fixed, as was done by Lilje \& Lahav (1991), who used results by BBKS. Thus, we impose that the number density of peaks in the filtered density field, that are higher than the threshold $v_{\mathrm{T}}$, equals the observed number density of Abell clusters in the universe, $n_{p k}\left(v_{\mathrm{T}}\right) \approx 10^{-5} h^{3} \mathrm{Mpc}^{-3}$ (see, for example, Abell 1958; Bahcall 1988); that is,

$n_{p k}\left(\nu_{\mathrm{T}}\right)=\int_{v_{\mathrm{T}}}^{\infty} \mathcal{N}_{p k}(v) \mathrm{d} v=10^{-5} h^{3} \mathrm{Mpc}^{-3}$,

where $\mathcal{N}_{p k}(v)$ is the differential number density of peaks with height $v$, given by Eqs. (4.3)-(4.6) of the work by BBKS. Thus,

$v_{\mathrm{T}}=2.95$.

In this study, instead of working with clusters of different height of their maxima, we deal with the average profile of density. Hence, we use the average value of $v$ weighted by the number density of peaks at a given height; that is,

$\langle v\rangle=\frac{\int_{v_{\mathrm{T}}}^{\infty} v \mathcal{N}_{p k}(v) \mathrm{d} v}{\int_{v_{\mathrm{T}}}^{\infty} \mathcal{N}_{p k}(v) \mathrm{d} v}$.

Assuming $v_{\mathrm{T}}=2.95$, we obtain

$\langle v\rangle=3.32$.

Hence, using the results (60) and (66) in Eq. (52),

$\langle\chi\rangle=8.45 \times 10^{-1}$.

Finally, once $\sigma_{0}$ is known, the density field, $\langle D(x)\rangle$, is fully determined. Our result for the rms fluctuations of the density field is (see Eq. (48))

$\sigma_{0}=9.71 \times 10^{-1}$.

\subsection{Numerical results}

In this subsection, the non-linear collapse of Newtonian spherical mass shells in a CDM average cluster has been calculated from $z_{\mathrm{I}}=1000$. This redshift corresponds to a time $t_{\mathrm{I}}=$ $4.706 \times 10^{5} \mathrm{yr}$ since the beginning of the Universe; this value has been calculated from (see Eq. (13)):

$t=\int_{0}^{a / a_{0}} \frac{\mathrm{d}\left(a^{\prime} / a_{0}\right)}{\left(a^{\prime} / a_{0}\right) H\left(a^{\prime} / a_{0}\right)}$,

with $a=a_{\mathrm{I}}=a_{0} /\left(1+z_{\mathrm{I}}\right)$ and assuming Eqs. (32)-(35), with $\Omega_{\mathrm{m} 0}=0.27$ and $H_{0}=71 \mathrm{~km} \mathrm{~s}^{-1} \mathrm{Mpc}^{-1}$. Equation (69) gives an age of the universe (at $a=a_{0}$ ) of $1.368 \times 10^{10} \mathrm{yr}$.

We have assumed an average density profile with a height at its maximum that is equal to that of the average peak (see Eqs. (49), (53)-(60), (62), (66)-(68)). At $z_{\mathrm{I}}=1000$, such a cluster has an overdensity at its maximum given by (see Eqs. (39), (45), (47) (66) and (68))

$\left\langle\delta\left(x=0, a_{\mathrm{I}}\right)\right\rangle=\frac{\delta_{\mathrm{g}}\left(a_{\mathrm{I}}\right)}{\delta_{\mathrm{g}}\left(a_{0}\right)}\langle v\rangle \sigma_{0}=4.77 \times 10^{-3}$.

Equation (27) has been integrated for shells enclosing different masses. Equations (28) and (29) have been used as boundary conditions. In Eq, (29), $f_{\mathrm{g}}\left(a_{\mathrm{I}}\right)$ is given by Eq. (46). For each mass shell $i$, we use $\left\langle\Delta_{i}\left(a_{\mathrm{I}}\right)\right\rangle$ instead of taking the average density profile $\Delta_{i}\left(a_{\mathrm{I}}\right)$; that is, we have taken an average density contrast $\left\langle\delta\left(x, a_{\mathrm{I}}\right)\right\rangle$ in Eq. (18) assuming the average density profile cited above. 
At $z_{\mathrm{I}}$, a shell $i$, which encloses a mass $\mathcal{M}_{i}$, has a proper radius given by Eq. (21) and a proper velocity given by Eqs. (22) and (24). For our average cluster, we have seen that this proper velocity should decrease until taking a value equal to zero at some $z_{i}^{\mathrm{TA}}$ if $\mathcal{M}_{i}<8.13 \times 10^{14} \mathcal{M}_{\odot}$ (i.e., the shell should be able to reach $\dot{r}_{i}\left(z_{i}^{\mathrm{TA}}\right)=0$ ). This redshift is known as the turnaround (TA) redshift for the shell $i$. At $z_{i}^{\mathrm{TA}}$, the shell radius is maximum and is named the turn-around radius, $r_{i}^{\mathrm{TA}}$. This redshift, $z_{i}^{\mathrm{TA}}$, corresponds to a time $t_{i}^{\mathrm{TA}}$ given by Eq. (69) with $a=$ $a_{0} /\left(1+z_{\mathrm{I}}^{\mathrm{TA}}\right)$. From $z_{i}^{\mathrm{TA}}$, the radius of the shell would begin to decrease. Finally, the collapse of the shell would end when a virialized structure with a virial radius, $r_{i}^{\mathrm{VIR}}$, is formed at $z_{i}^{\mathrm{VIR}}$ which fulfills $t_{i}^{\mathrm{VIR}} \approx 2 t_{i}^{\mathrm{TA}}$. In this work, the virial radius is estimated as was performed by Membrado \& Aguerri (2004); i.e. by imposing the conservation of the energy of the shell $i$ between $z_{i}^{\mathrm{TA}}$ and $z_{i}^{\mathrm{VIR}}$. At the turn-around point, it is assumed that the shell $i$ encloses a mass sphere of constant density, and at the virial redshift, a singular isothermal sphere is taken. At $z_{i}^{\mathrm{TA}}$, the kinetic energy of the shell $i$ is zero, while at $z_{i}^{\mathrm{VIR}}$, its kinetic energy can be calculated imposing the virial theorem under steady conditions. Thus, the relation between $r_{i}^{\mathrm{TA}}$ and $r_{i}^{\mathrm{VIR}}$ fulfills

$1=\frac{6}{5}\left[2-\frac{\zeta\left(z_{i}^{\mathrm{TA}}\right)}{\rho_{i}^{\mathrm{TA}}}\right]\left(\frac{r_{i}^{\mathrm{VIR}}}{r_{i}^{\mathrm{TA}}}\right)+\frac{5}{3}\left[\frac{\zeta\left(z_{i}^{\mathrm{VIR}}\right)}{\rho_{i}^{\mathrm{TA}}}\right]\left(\frac{r_{i}^{\mathrm{VIR}}}{r_{i}^{\mathrm{TA}}}\right)^{3}$,

where

$$
\begin{aligned}
\zeta(z) & =\left[\bar{\rho}_{\gamma}(z)+\bar{\rho}_{\Lambda}\right]+3\left[\bar{p}_{\gamma}(z)+\bar{p}_{\Lambda}\right], \\
\rho_{i}^{\mathrm{TA}} & =\frac{\bar{\rho}_{\mathrm{m}}\left(z_{I}\right)\left[1+\Delta_{i}\left(z_{\mathrm{I}}\right)\right]}{A_{i}^{3}\left(z_{i}^{\mathrm{TA}}\right)} .
\end{aligned}
$$

However, this does not occur for all shells. As said above, we have found that there is a limiting shell enclosing a mass for our average cluster

$$
\left\{\mathcal{M}^{\mathrm{VIR}}\right\}_{\mathrm{MAX}} \approx 8.13 \times 10^{14} M_{\odot},
$$

which is able to reach zero proper velocity; therefore, $\left\{\mathcal{M}^{\mathrm{VIR}}\right\}_{\text {MAX }}$ is the maximum mass of an average cluster that can virialize. This is a consequence of the repulsive effect of the cosmological constant. If a shell encloses a mass $\mathcal{M}_{i}>\left\{\mathcal{M}^{\mathrm{VIR}}\right\}_{\mathrm{MAX}}$, it reaches zero proper acceleration at certain redshift, $z_{i}^{\mathrm{ZA}}$. The radius of the shell at $z_{i}^{\mathrm{ZA}}$ is denoted by $r_{i}^{\mathrm{ZA}}$. For $z<z_{i}^{\mathrm{ZA}}$, the proper velocity continues to be positive, and the shell keeps expanding without reaching a turn-around radius.

The horizontal axis of Fig. 1 shows masses, $\mathcal{M}$, enclosed by shells of the average cluster. The lines in Fig. 1 show redshifts at which these mass shells reach their turn-around points, $z_{\mathcal{M}}^{\mathrm{TA}}$, their virialization, $z_{\mathcal{M}}^{\mathrm{VIR}}$, and their zero acceleration points, $z_{\mathcal{M}}^{\mathrm{ZA}}$.

Figure 1 indicates that shells enclosing masses greater than $8.13 \times 10^{14} M_{\odot}$ will never virialize. The dashed line of this figure shows redshifts at which those shells reach zero acceleration; so those shells will move away from the cluster center with a positive acceleration at smaller redshifts.

In this work, $\left[\mathcal{M}^{\mathrm{VIR}}\right]_{0}$ represents the mass of the virialized core of the cluster at present; $\left[\mathcal{M}^{\mathrm{TA}}\right]_{0}$ is the mass enclosed by the shell that is reaching the turn-around point at present; and $\left[\mathcal{M}^{\mathrm{ZA}}\right]_{0}$ corresponds to the mass inside the shell having zero proper acceleration at present. From Fig. 1, we see that $\left[\mathcal{M}^{\mathrm{VIR}}\right]_{0}=3.47 \times 10^{14} M_{\odot},\left[\mathcal{M}^{\mathrm{TA}}\right]_{0}=6.76 \times 10^{14} M_{\odot}$, and $\left[\mathcal{M}^{\mathrm{ZA}}\right]_{0}=1.10 \times 10^{15} M_{\odot}$. Therefore, for the average cluster, $\left[\mathcal{M}^{\mathrm{TA}}\right]_{0}=1.95\left[\mathcal{M}^{\mathrm{VIR}}\right]_{0}$, and $\left[\mathcal{M}^{\mathrm{ZA}}\right]_{0}=1.63\left[\mathcal{M}^{\mathrm{TA}}\right]_{0}$.

Thus, shells enclosing masses fulfilling $\mathcal{M} \leq\left[\mathcal{M}^{\mathrm{VIR}}\right]_{0}$ are virialized at present. Those with $\left[\mathcal{M}^{\mathrm{VIR}}\right]_{0}<\mathcal{M} \leq\left[\mathcal{M}^{\mathrm{TA}}\right]_{0}$

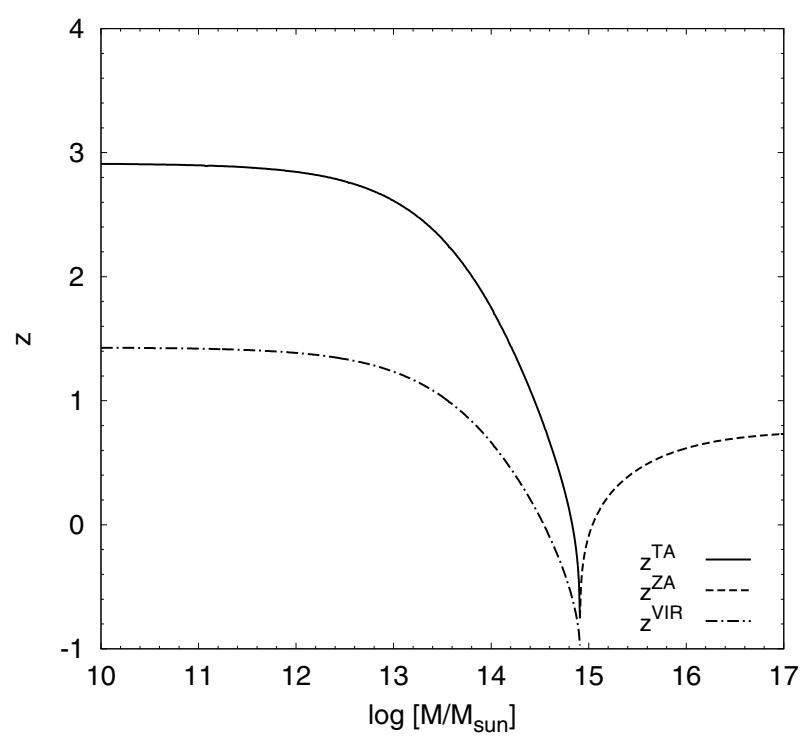

Fig. 1. Zero proper velocity redshift, $z^{\mathrm{TA}}$, zero proper acceleration redshift, $z^{\mathrm{ZA}}$, and virialization redshift, $z^{\mathrm{VIR}}$, for masses enclosed by shells of the average cluster.

have reached their turn-around radii and virializes in the future. Shells fulfilling $\left[\mathcal{M}^{\mathrm{TA}}\right]_{0}<\mathcal{M} \leq\left\{\mathcal{M}^{\mathrm{VIR}}\right\}_{\mathrm{MAX}}$ will reach a turnaround radius in the future and will virialize; the shell enclosing a mass equal to $\left\{\mathcal{M}^{\mathrm{VIR}}\right\}_{\text {MAX }}$ will reach its turn-around point at $z_{\left\{\mathcal{M}^{\mathrm{TIR}}\right\}_{\text {MAX }}}^{\mathrm{TA}}=-0.753$, and will virialize at $z_{\left\{\mathcal{M}^{\mathrm{VIR}}\right\}_{\text {MAX }}}^{\mathrm{VR}}=-0.973$. Those shells with $\left\{\mathcal{M}^{\mathrm{VIR}}\right\}_{\text {MAX }}<\mathcal{M} \leq\left[\mathcal{M}^{\mathrm{ZA}}\right]_{0}$ show negative proper accelerations at present but will fulfill $\ddot{r}=0$ in the future and will never reach a turn-around radius. Finally, shells enclosing masses fulfilling $\left[\mathcal{M}^{\mathrm{ZA}}\right]_{0}<\mathcal{M}$ show positive proper acceleration and keep expanding.

Figure 1 indicates that the first shell, for our average cluster model, that reached its turn-around radius did so at $\left\{z^{\mathrm{TA}}\right\}_{\mathrm{MAX}}=$ 2.91. This figure also shows that the first shell that virialized did so at redshift $\left\{z^{\mathrm{VIR}}\right\}_{\text {MAX }}=1.43$.

In Fig. 1, it is also shown that shells enclosing masses $\mathcal{M} \gg$ $\left\{\mathcal{M}^{\mathrm{VIR}}\right\}_{\text {MAX }}$ reached $\ddot{r}=0$ at redshifts close to the asymptotic value $\left\{z^{\mathrm{ZA}}\right\}_{\text {MAX }}=0.755$. This asymptotic redshift corresponds to $z_{U}^{\mathrm{ZA}}$ at which $\ddot{a}=0$ (see Eq. (15), assuming $\Omega_{\mathrm{m} 0}=0.27$ and $\Omega_{\Lambda}=0.73$ ). Hence, $\left\{z^{\mathrm{ZA}}\right\}_{\mathrm{MAX}}$ is the redshift at which the repulsive effect of the cosmological constant equals the gravitational strength due to the background non-relativistic matter. According to what was also stated in Sect. $3,\left\{z^{Z A}\right\}_{\text {MAX }}$ is the redshift, $z_{\mathrm{c}}$, at which background non-relativistic matter reaches the critical radius (see Eq. (12) with $\Omega_{\mathrm{m} 0}=0.27$ and $\Omega_{\Lambda}=0.73$ ).

At each $z$, one can find one shell that virializes, a second shell that reaches its turn-around radius, and a third one which is at its zero acceleration point. In Fig. 2, we show the turn-around radius, $\left[r^{\mathrm{TA}}\right]_{z}$, the zero acceleration radius, $\left[r^{\mathrm{ZA}}\right]_{z}$, and the virial radius, $\left[r^{\mathrm{VIR}}\right]_{z}$, of these shells as a function of the redshift $z$. In this figure, the maximum turn-around radius which the average cluster is able to reach can be seen; its value is $\left\{r^{\mathrm{TA}}\right\}_{\mathrm{MAX}}=9.91 \mathrm{Mpc}$ and is reached in the future at $z=z_{\left\{\mathcal{M}^{\mathrm{TIR}}\right\}_{\mathrm{MAX}}}^{\mathrm{TA}}=-0.753$. In the same figure, it is shown that the maximum virial radius of the average cluster is $\left\{r^{\mathrm{VIR}}\right\}_{\mathrm{MAX}}=2.84 \mathrm{Mpc}$ which will be reached at $z=z_{\left\{\mathcal{M}^{\mathrm{VIR}}\right\}_{\text {MAX }}}=-0.973$.

Figure 2 shows that $\left[r^{\mathrm{VIR}}\right]_{0}=1.50 \mathrm{Mpc},\left[r^{\mathrm{TA}}\right]_{0}=6.85 \mathrm{Mpc}$, and $\left[r^{\mathrm{ZA}}\right]_{0}=10.87 \mathrm{Mpc}$. $\left[r^{\mathrm{VIR}}\right]_{0}$ is the radius of the mass shell that virializes at present; $\left[r^{\mathrm{TA}}\right]_{0}$ represents the radius of the shell that reaches the turn-around point at present; and $\left[r^{\mathrm{ZA}}\right]_{0}$ indicates 


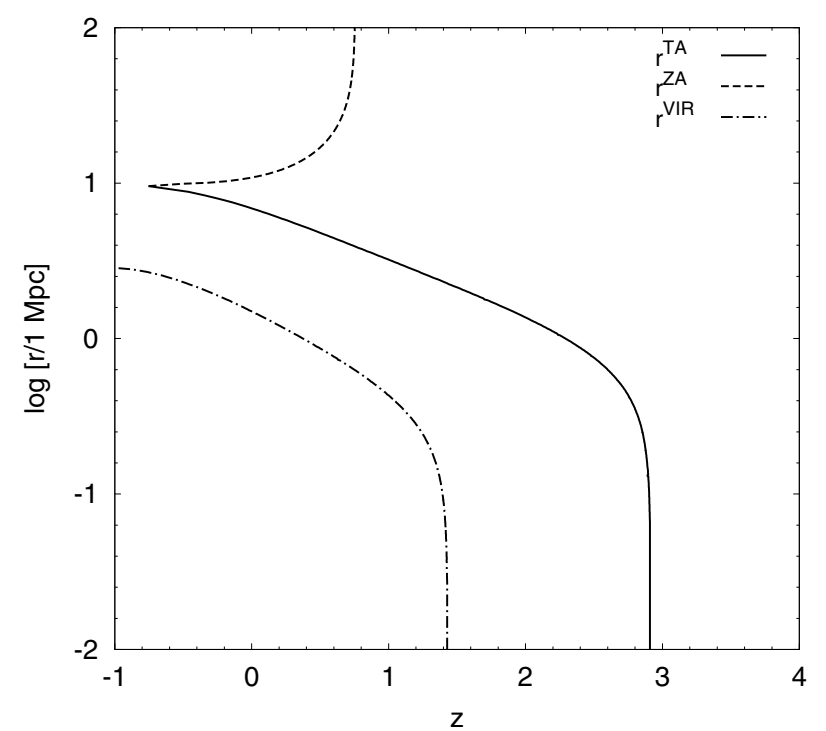

Fig. 2. For each redshift $z$ : the continuous line represents the turnaround radius of the shell of the average cluster that reaches its turnaround point at that redshift; the dot-dashed line shows the virial radius of the shell which virializes at $z$; finally, the dashed line indicates the zero acceleration radius of the shell that reaches its zero acceleration point at $z$.

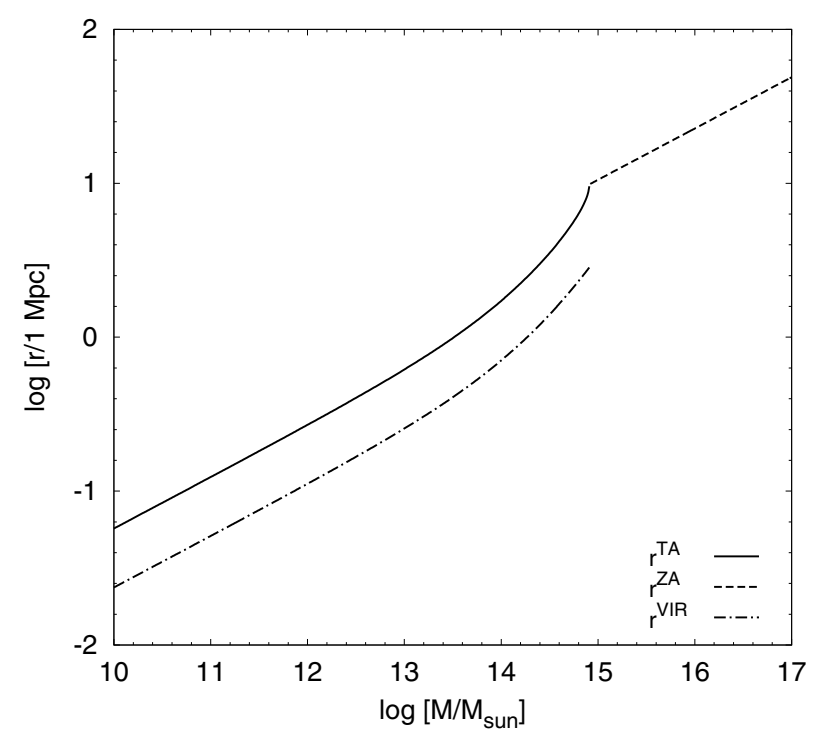

Fig. 3. Turn-around radius, $r^{\mathrm{TA}}$, zero proper acceleration radius, $r^{\mathrm{ZA}}$, and virial radius, $r^{\mathrm{VIR}}$, for masses enclosed by shells of the average cluster.

the radius of the shell with zero proper acceleration at present. Figure 2 also shows $\left\{z^{\mathrm{TA}}\right\}_{\text {MAX }}$ and $\left\{z^{\mathrm{VIR}}\right\}_{\text {MAX }}$.

It should also be said that similar figures to Figs. 1 and 2 are obtained when the filtering scale, $R_{\mathrm{f}}$, is taken in the order of galaxy scales or galaxy group scales. This means that the cosmological constant also imposes mass limits on galaxies and on the virialized core of galaxy groups.

In Fig. 3, we show radii reached for shells enclosing different masses $\mathcal{M}$ at their turn-around redshifts, $r_{\mathcal{M}}^{\mathrm{TA}}$, when they virialize, $r_{\mathcal{M}}^{\mathrm{VIR}}$, and at their zero proper acceleration redshifts, $r_{\mathcal{M}}^{\mathrm{ZA}}$. In this figure, $r_{\left\{\mathcal{M}^{\mathrm{VIR}}\right\}_{\mathrm{MAX}}}^{\mathrm{VIR}} \equiv\left\{r^{\mathrm{VIR}}\right\}_{\mathrm{MAX}}$ and $r_{\left\{\mathcal{M}^{\mathrm{VIR}}\right\}_{\mathrm{MAX}}^{\mathrm{TA}}} \equiv\left\{r^{\mathrm{TA}}\right\}_{\mathrm{MAX}}$ can also be seen.

The numerical results shown in Figs. 1 and 2 for shells reaching virialization or a turn-around point at different redshifts can be fitted by analytical expressions. Here, we show some expressions for redshifts $0 \leq z \leq z_{3 / 4}=0.266$, where $\Omega_{\mathrm{m}}\left(z_{3 / 4}\right)=$ $(3 / 4) \Omega_{\Lambda}$. Thus,

$$
\begin{aligned}
& \left(\frac{\left[\mathcal{M}^{\mathrm{VIR}}\right]_{z}}{10^{14} \mathcal{M}_{\odot}}\right) \approx 1.03(1+z)^{1.76}\left(\frac{\left[r^{\mathrm{VIR}}\right]_{z}}{1 \mathrm{Mpc}}\right)^{3}, \\
& \left(\frac{\left[\mathcal{M}^{\mathrm{TA}}\right]_{z}}{10^{15} \mathcal{M}_{\odot}}\right) \approx 2.10(1+z)^{1.62}\left(\frac{\left[r^{\mathrm{TA}}\right]_{z}}{10 \mathrm{Mpc}}\right)^{3} .
\end{aligned}
$$

It should be said that the difference with respect to the numerical results is smaller than $0.9 \%$ for Eq. (75) and smaller than $0.7 \%$, for (76).

The turn-around radius has often been used to estimate the mass of a gravitationally bound system. Lynden-Bell (1981) and Sandage (1986) derived an expression relating both magnitudes at present; in the simplest case of spherical symmetry with $\Lambda=$ 0 , they showed that $\left[r^{\mathrm{TA}}\right]_{0}$ depends only on the total mass of a the cluster, $\mathcal{M}_{\mathrm{T}}$, and on the age of the Universe, $t_{0}$; i.e.,

$\mathcal{M}_{\mathrm{T}}=\frac{\pi^{2}}{G} \frac{\left(\left[r^{\mathrm{TA}}\right]_{0}\right)^{3}}{t_{0}^{2}}$.

Karanchentsev et al. (2002, 2006, 2009), Karanchentsev \& Kashibadze (2006), investigated the Local Group and other proximate groups and obtained masses derived from the LyndenBell and Sandage estimator. For each studied group, they calculated the quotient between its estimated mass and its virial mass; the mean value of these quotients was 0.6 (see, for example, Karanchentsev 2005). However, when the cosmological constant was included in the calculations, such mean values approached unity.

For a flat universe with $\Lambda \neq 0$, Eq. (77) reads as (see, for example, Karachentsev \& Novasona 2010):

$\mathcal{M}_{\mathrm{T}}=\frac{\pi^{2}}{8 G}\left(\left[r^{\mathrm{TA}}\right]_{0}\right)^{3} \frac{H_{0}^{2}}{f^{2}\left(\Omega_{\mathrm{m} 0}\right)}$,

where

$f\left(\Omega_{\mathrm{m} 0}\right)=\frac{1}{1-\Omega_{\mathrm{m} 0}}-\frac{\Omega_{\mathrm{m} 0}}{2\left(1-\Omega_{\mathrm{m} 0}\right)^{3 / 2}} \operatorname{arccosh}\left[\frac{2}{\Omega_{\mathrm{m} 0}}-1\right]$.

Hence, assuming $H_{0}=71 \mathrm{kms}^{-1} \mathrm{kpc}^{-1}$ and $\Omega_{\mathrm{m} 0}=0.27$, Eqs. (78) and (79) indicate that

$\left(\frac{\mathcal{M}_{\mathrm{T}}}{10^{15} \mathcal{M}_{\odot}}\right)=2.16\left(\frac{\left[r^{\mathrm{TA}}\right]_{0}}{10 \mathrm{Mpc}}\right)^{3}$.

As can be seen, Eq. (76) at $z=0$ and Eq. (80) are rather similar; the difference between both equations is smaller than $2.6 \%$. Thus, $\mathcal{M}_{\mathrm{T}}$ in Eq. (80) could correspond to $\left[\mathcal{M}^{\mathrm{TA}}\right]_{0}$.

Now, we can have a look at the results by Rines et al. (2003) that relate to virial and turn-around data of the clusters studied in the CAIRNS (Cluster and Infall Region Nearby Survey) project. When their data are averaged (the large clusters A539 and A1656, and the small cluster A194 are not considered) and $H_{0}=71 \mathrm{~km} \mathrm{~s}^{-1} \mathrm{Mpc}^{-1}$ is assumed, $\left\langle r^{\mathrm{VIR}}\right\rangle=1.52 \mathrm{Mpc}$, $\left\langle r^{\mathrm{TA}}\right\rangle=7.02 \mathrm{Mpc},\left\langle\mathcal{M}^{\mathrm{VIR}}\right\rangle=4.15 \times 10^{14} \mathcal{M}_{\odot}$, and $\left\langle\mathcal{M}^{\mathrm{TA}}\right\rangle=$ $7.16 \times 10^{14} \mathcal{M}_{\odot}$ are obtained. These average values lead to

$\left(\frac{\left\langle\mathcal{M}^{\mathrm{VIR}}\right\rangle}{10^{14} \mathcal{M}_{\odot}}\right)=1.18\left(\frac{\left\langle r^{\mathrm{VIR}}\right\rangle}{1 \mathrm{Mpc}}\right)^{3}$

$\left(\frac{\left\langle\mathcal{M}^{\mathrm{TA}}\right\rangle}{10^{15} \mathcal{M}_{\odot}}\right)=2.07\left(\frac{\left\langle r^{\mathrm{TA}}\right\rangle}{10 \mathrm{Mpc}}\right)^{3}$ 
Comparing Eqs. (75) at $z=0$ and (81) for the virialized core, we see that the difference is about $12 \%$. However, for the turnaround surface, such a difference is smaller than $1.5 \%$ (see Eqs. (76) at $z=0$ and (82)).

From Fig. 2, we have seen that, at each $z$, there could be a shell reaching its turn-around point and another shell which is virializing. The following expression relates the turn-around radius of the shell which reaches the turn-around point at redshift $z$ with the virial radius of the other shell that virializes at the same $z$, for $0 \leq z \leq z_{3 / 4}$ :

$\left[r^{\mathrm{VIR}}\right]_{z} \approx 6.97 \times 10^{-1}(1+z)^{-0.57}\left[\left[r^{\mathrm{TA}}\right]_{z}-\left[r^{\mathrm{TA}}\right]_{\left\{z^{\mathrm{VIR}}\right\}_{\mathrm{MAX}}}\right]^{1 / 2}$

In Eq. (83), $\left[r^{\mathrm{TA}}\right]_{\left\{z^{\mathrm{VIR}}\right\}_{\mathrm{MAX}}}=2.27 \mathrm{Mpc}$ is the turn-around radius of the shell that is reached at the greatest redshift at which a shell can virialize, which is at $\left\{z^{\mathrm{VIR}}\right\}_{\mathrm{MAX}}=1.43$ (see Fig. 1 or Fig. 2). The term $\left[\left[r^{\mathrm{TA}}\right]_{z}-\left[r^{\mathrm{TA}}\right]_{\left\{z^{\mathrm{VIR}}\right\}_{\mathrm{MAX}}}\right]^{1 / 2}$ is used in Eq. (83) because at $z_{5 / 4} \leq z \leq\left\{z^{\mathrm{VIR}}\right\}_{\mathrm{MAX}}$

$\left[r^{\mathrm{VIR}}\right]_{z} \approx 7.30 \times 10^{-1}(1+z)^{-0.72}\left[\left[r^{\mathrm{TA}}\right]_{z}-\left[r^{\mathrm{TA}}\right]_{\left\{z^{\mathrm{VIR}}\right\}_{\mathrm{MAX}}}\right]^{1 / 2}$,

where $\Omega_{\mathrm{m}}\left(z_{5 / 4}\right)=(5 / 4) \Omega_{\Lambda}$, so $z_{5 / 4}=0.501$. As can be seen from Eq. (84), $\left[r^{\mathrm{VIR}}\right]_{\left\{z^{\mathrm{VIR}}\right\}_{\text {MAX }}}=0$ (see Fig. 2). The difference with respect to the numerical results is smaller than $0.2 \%$ for Eq. (83) and smaller than $0.6 \%$ for (84).

Now, we could calculate the quotient between the radius of the shell, for the average cluster, which reaches its turn-around point at present and the radius of the shell which virializes at present. Thus,

$\left[r^{\mathrm{TA}}\right]_{0}=4.57\left[r^{\mathrm{VIR}}\right]_{0}$.

With respect to the quotient between the mass enclosed by the shell that reaches its turn-around point and the mass enclosed by shell that virializes, we have found

$\left[\mathcal{M}^{\mathrm{TA}}\right]_{0}=1.95\left[\mathcal{M}^{\mathrm{VIR}}\right]_{0}$.

Equations (85) and (86) can be compared with the results by Rines \& Diaferio (2006), who used the Fourth Data Release of the Sloan Digital Sky Survey (SDSS). Their results averaged over all clusters were

$\left\langle r^{\mathrm{TA}}\right\rangle=4.75\left\langle r^{\mathrm{VIR}}\right\rangle$,

$\left\langle\mathcal{M}^{\mathrm{TA}}\right\rangle=1.97\left\langle\mathcal{M}^{\mathrm{VIR}}\right\rangle$.

The difference between Eq. (85) and (87) is about 4\%, while it is $1 \%$ between (86) and (88).

Hence, Eqs. (76) at $z=0$ and (86) derived from our theoretical average cluster agree with Eqs. (82) and (88) coming from observational measurements, which are averaged over clusters. However, Eq. (75) at $z=0$ and (85), which deal with virial radii, must only be considered as estimations; the difference between these equations and (81) and (87) could be due to the simple model we have used to determine the virial radii.

From Fig. 2, we know that at each $z$, there could be a shell reaching its turn-around point and another shell that shows zero proper acceleration. The relation between the turn-around radius of the first shell and the zero proper acceleration radius of the second can be shown using the following analytical expression,

$\left[r^{\mathrm{ZA}}\right]_{z} \approx 1.88 \times 10^{1} G_{z}\left[\left[r^{\mathrm{TA}}\right]_{z}-\left[r^{\mathrm{TA}}\right]_{\{z \mathrm{ZA}\}_{\mathrm{MAX}}}\right]^{-1 / 2}$, where

$G_{z}= \begin{cases}9.71 \times 10^{-1}(1+z)^{-0.31}, & z_{5 / 4} \leq z \leq\left\{z^{\mathrm{ZA}}\right\}_{\mathrm{MAX}} \\ 1.00(1+z)^{-0.39}, & 0 \leq z<z_{5 / 4}\end{cases}$

and $\left[r^{\mathrm{TA}}\right]_{\{z \mathrm{ZA}\}_{\text {MAX }}}=3.89 \mathrm{Mpc}$.

Finally, it should also be said that the zero acceleration radii of shells fulfilling $\mathcal{M}>\mathcal{M}_{\mathrm{MAX}}^{\mathrm{VIR}}$ show the behavior

$\left[\mathcal{M}^{\mathrm{ZA}}\right]_{z}=\frac{\Lambda c^{2}}{3 G}\left(\left[r^{\mathrm{ZA}}\right]_{z}\right)^{3}, z_{\left\{\mathcal{M}^{\mathrm{VIR}}\right\}_{\mathrm{MAX}}}^{\mathrm{TA}}<z \leq\left\{z^{\mathrm{ZA}}\right\}_{\mathrm{MAX}}$,

which agrees with Eq. (7).

\subsection{Comparison with the case $\Lambda=0$}

This subsection compares the model presented in previous subsections with a similar model in which the cosmological constant is zero in a flat universe. Hence, we assume $\bar{\rho}_{\Lambda}=\bar{p}_{\Lambda}=0$.

With respect to the growing solution at the expanding parameter $a_{\mathrm{I}}$ of the linearized Eq. (31) for the growth of matter perturbations, we find

$\frac{\delta_{\mathrm{g}}\left(a_{\mathrm{I}}\right)}{\delta_{\mathrm{g}}\left(a_{0}\right)}=1.03 \times 10^{-3}$,

$\left.\frac{a_{\mathrm{I}}}{\delta_{\mathrm{g}}\left(a_{\mathrm{I}}\right)} \frac{\mathrm{d} \delta_{\mathrm{g}}}{\mathrm{d} a}\right|_{a_{\mathrm{I}}}=9.67 \times 10^{-1}$.

The spectral parameters, which fix the average density field, $\langle D(x)\rangle$, are found to be

$R_{\star}=3.85 h^{-1} \mathrm{Mpc}$,

$\gamma=7.31 \times 10^{-1}$

$P_{0}=4.86 \times 10^{4} h^{-4} \mathrm{Mpc}^{4}$,

$v_{\mathrm{T}}=3.20$,

$\langle v\rangle=3.54$

$\langle\chi\rangle=6.06 \times 10^{-1}$

$\sigma_{0}=6.21 \times 10^{-1}$

(a filtering scale, $R_{\mathrm{f}}=3 h^{-1} \mathrm{Mpc}$, and $H_{0}=71 \mathrm{~km} \mathrm{~s}^{-1} \mathrm{Mpc}^{-1}$ have been assumed).

As in the previous subsection, the non-linear collapse of Newtonian spherical mass shells in an average cluster has been studied from $z_{\mathrm{I}}=1000$. At this redshift, this cluster has an overdensity at its maximum,

$\left\langle\delta\left(x=0, a_{\mathrm{I}}\right)\right\rangle=\frac{\delta_{\mathrm{g}}\left(a_{\mathrm{I}}\right)}{\delta_{\mathrm{g}}\left(a_{0}\right)}\langle v\rangle \sigma_{0}=2.26 \times 10^{-3}$.

Results of this model are shown in Figs. 4 and 5. In theses figures, wide lines represent results assuming $\Lambda=0$ in a flat universe, while narrow lines are those obtained in Sect. 4.4 for our $\Lambda$ CDM model.

From Fig. 4, we see that, for $\Lambda=0$, turn-around points are later than $z \approx 1.06$ and that virialization appears at redshifts smaller than 0.3 . These results could be in conflict with the observed high redshift clusters (see, for example, Gladders \& Yee 2005; Goto et al. 2008; Wen et al. 2009; Hao et al. 2010; Wen \& Han 2011).

In Fig. 4 , it is also shown that, at present, $\left[\mathcal{M}^{\mathrm{VIR}}(\Lambda=0)\right]_{0}=$ $1.51 \times 10^{14} M_{\odot}$ and $\left[\mathcal{M}^{\mathrm{TA}}(\Lambda=0)\right]_{0}=7.94 \times 10^{14} M_{\odot}$. These results indicate that

$\frac{\left[\mathcal{M}^{\mathrm{TA}}(\Lambda=0)\right]_{0}}{\left[\mathcal{M}^{\mathrm{VIR}}(\Lambda=0)\right]_{0}}=5.26$ 


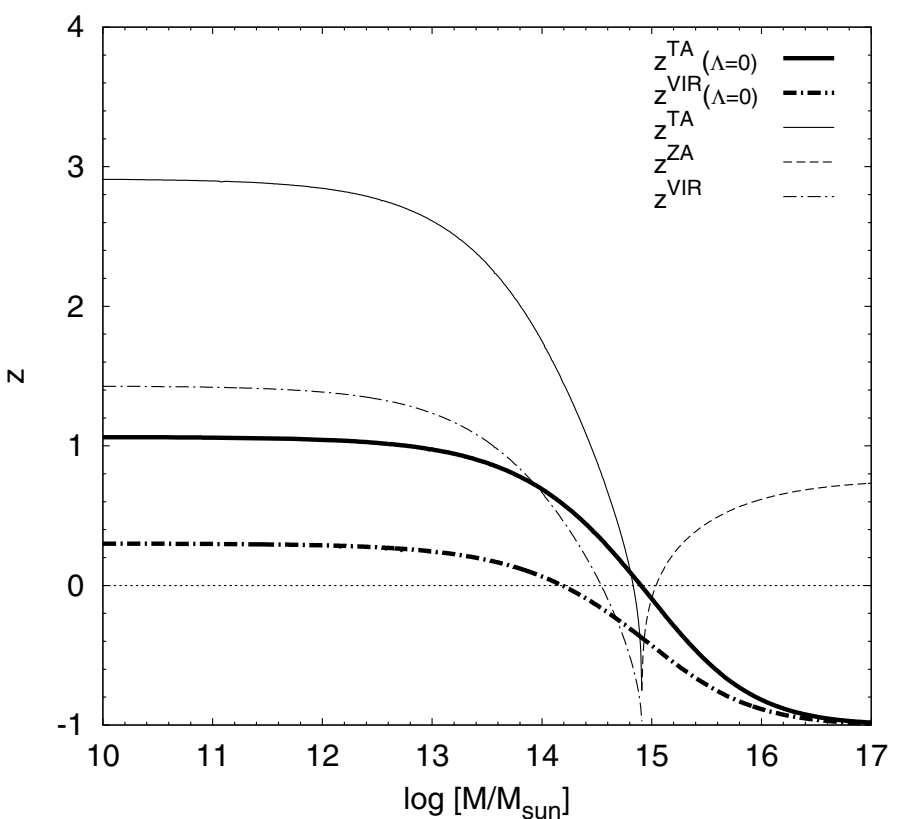

Fig. 4. Wide lines $(\Lambda=0$ model $)$ : zero proper velocity redshift, $z^{\mathrm{TA}}(\Lambda=$ 0 ), and virialization redshift, $z^{\mathrm{VIR}}(\Lambda=0)$, for masses enclosed by shells. Narrow lines $\left(\Lambda \mathrm{CDM}\right.$ model shown in Fig. 1): $z^{\mathrm{TA}}, z^{\mathrm{ZA}}$, and $z^{\mathrm{VIR}}$ for masses enclosed by shells.

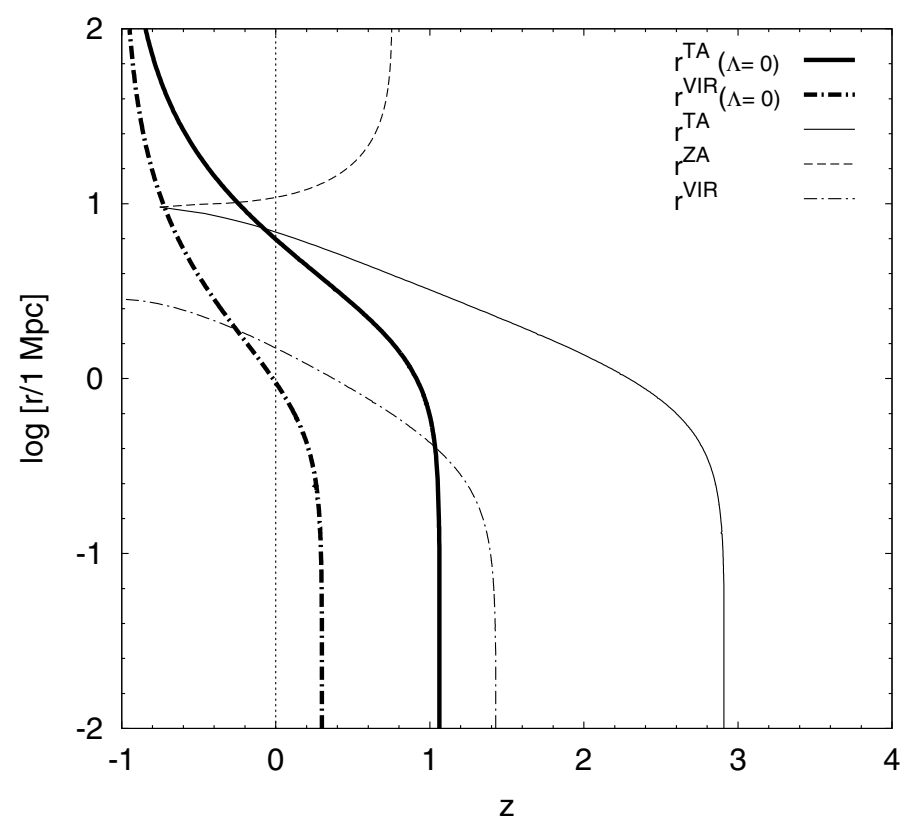

Fig. 5. Wide lines $(\Lambda=0$ model): the continuous line represents the turn-around radius of the shell that reaches its turn-around point at redshift $z$; the dot-dashed line shows the virial radius of the shell that virializes at $z$. Narrow lines ( $\Lambda \mathrm{CDM}$ model shown in Fig. 2): $r^{\mathrm{TA}}, r^{\mathrm{ZA}}$, and $r^{\mathrm{VIR}}$ as a function of $z$.

The quotient expressed by Eq. (102) is 2.67 times greater than that shown in Eq. (88) and derived from the work by Rines \& Diaferio (2006). According with this result, we can say that our $\Lambda=0$ model does not agree with observations.

At present, Fig. 5 indicates that $\left[r^{\mathrm{VIR}}(\Lambda=0)\right]_{0}=0.943 \mathrm{Mpc}$ and $\left[r^{\mathrm{TA}}(\Lambda=0)\right]_{0}=6.24 \mathrm{Mpc}$. Therefore,

$$
\frac{\left[r^{\mathrm{TA}}(\Lambda=0)\right]_{0}}{\left[r^{\mathrm{VIR}}(\Lambda=0)\right]_{0}=6.62 \text {. }}
$$

As happened with Eq. (102), this quotient does not agree with that derived from the work by Rines \& Diaferio (2006) (see Eq. (87)).

The following relations are also obtained from our $\Lambda=$ 0 model:

$$
\begin{aligned}
\left(\frac{\left[\mathcal{M}^{\mathrm{VIR}}(\Lambda=0)\right]_{0}}{10^{14} \mathcal{M}_{\odot}}\right) & =1.80\left(\frac{\left[r^{\mathrm{VIR}}(\Lambda=0)\right]_{0}}{1 \mathrm{Mpc}}\right)^{3}, \\
\left(\frac{\left[\mathcal{M}^{\mathrm{TA}}(\Lambda=0)\right]_{0}}{10^{15} \mathcal{M}_{\odot}}\right) & =3.27\left(\frac{\left[r^{\mathrm{TA}}(\Lambda=0)\right]_{0}}{10 \mathrm{Mpc}}\right)^{3} .
\end{aligned}
$$

The relations shown by Eqs. (104) and (105) also differ from those derived from the work by Rines et al. (2003), as expressed by Eqs. (81) and (82). These results also confirm that $\Lambda=0$ model disagrees with observations.

\section{Discussion}

The time integration of Eq. (20), when radiation terms are neglected, is straightforward. Its value is

$\frac{1}{2} \dot{r}_{i}^{2}-\frac{G}{r_{i}} \mathcal{M}_{i}+\frac{2 \pi G}{3 c^{2}}\left[\bar{\rho}_{\Lambda}+3 \bar{p}_{\Lambda}\right] r_{i}^{2}=K_{i}$,

where $K_{i}$ is the total mechanical energy of the shell $i$. The parameter $K_{i}$ is conserved in the evolution of the shell $i$. From Eqs. (34) and (35), the radiation terms equal the cosmological constant terms at redshift

$1+z_{\gamma=\Lambda}=1.094 \times 10^{1}\left(\frac{\Omega_{\Lambda}}{0.73}\right)^{1 / 4}\left(\frac{H_{0}}{71 \mathrm{~km} \mathrm{~s}^{-1} \mathrm{Mpc}^{-1}}\right)^{1 / 2}$.

Hence, Eq. (106) can be used at redshifts $z \ll z_{\gamma=\Lambda}$.

As was shown in the previous section, a shell $i$ from our average cluster with a mass $\mathcal{M}_{i}>\left\{\mathcal{M}^{\mathrm{VIR}}\right\}_{\max } \approx 8.13 \times 10^{14} M_{\odot}$ is not able to reach a turn-around point. Besides at some $z_{i}^{\mathrm{ZA}}$, which corresponds to a time $t_{i}^{\mathrm{ZA}}$ (see Eq. (69)), this shell $i$ shows zero proper acceleration. Figure 1 shows that shells reach their zero proper acceleration radii at redshifts $z^{\mathrm{ZA}}<z_{U}^{\mathrm{ZA}}=0.755$. Hence, $\bar{\rho}_{\gamma}\left(z^{\mathrm{ZA}}\right) / \bar{\rho}_{\Lambda}<6.62 \times 10^{-4}\left(\Omega_{\Lambda}=0.73\right.$ and $H_{0}=71 \mathrm{~km} \mathrm{~s}^{-1} \mathrm{kpc}^{-1}$ have been assumed). Thus, for these shells, Eq. (106) is valid, and their evaluation at their corresponding $z_{i}^{\mathrm{ZA}}$ 's could be used to obtain their $K_{i}$ 's. That is,

$K_{i}=\frac{1}{2}\left(\dot{r}_{i}^{\mathrm{ZA}}\right)^{2}-\frac{G}{r_{i}^{\mathrm{ZA}}} \mathcal{M}_{i}+\frac{2 \pi G}{3 c^{2}}\left[\bar{\rho}_{\Lambda}+3 \bar{p}_{\Lambda}\right]\left(r_{i}^{\mathrm{ZA}}\right)^{2}$.

At times $t>t_{i}^{\mathrm{ZA}}$, these shells acquire positive acceleration, and at long enougth times, $t \gg t_{i}^{\mathrm{ZA}}$, their radii are so large that the matter gravitational term in Eq. (106) is negligible with respect to the cosmological constant term. Hence, at $t \gg t_{i}^{\mathrm{ZA}}$,

$\dot{r}_{i}=\left(\frac{8 \pi G \rho_{\Lambda}}{3 c^{2}}\right)^{1 / 2} r_{i}$.

Equation (109) was the argument given by Chernin et al. (2010) to solve the Hubble-Sandage paradox on the scale of $\sim 10 \mathrm{Mpc}$ (Sandage 1986, 1999) observed that the local expansion was similar to that due to the Hubble flow. In the case of a shell $i$, its proper velocity at $t \gg t_{i}^{\mathrm{ZA}}$ is $\dot{r}_{i}=H_{G C} r_{i}$ with $H_{G C}=$ $\left[\left(8 \pi G \rho_{\Lambda}\right) /\left(3 c^{2}\right)\right]^{1 / 2}$, while the velocity due to the Hubble flow is $H r_{i}, H$ being the Hubble function at $t$.

However, the asymptotic behavior shown in Eq. (109) has not been reached by any shell at present. To note this, we can have a look at Eq. (106) and to our average cluster at present. 
M. Membrado and A. F. Pacheco: Effects of the cosmological constant on cold dark matter clusters

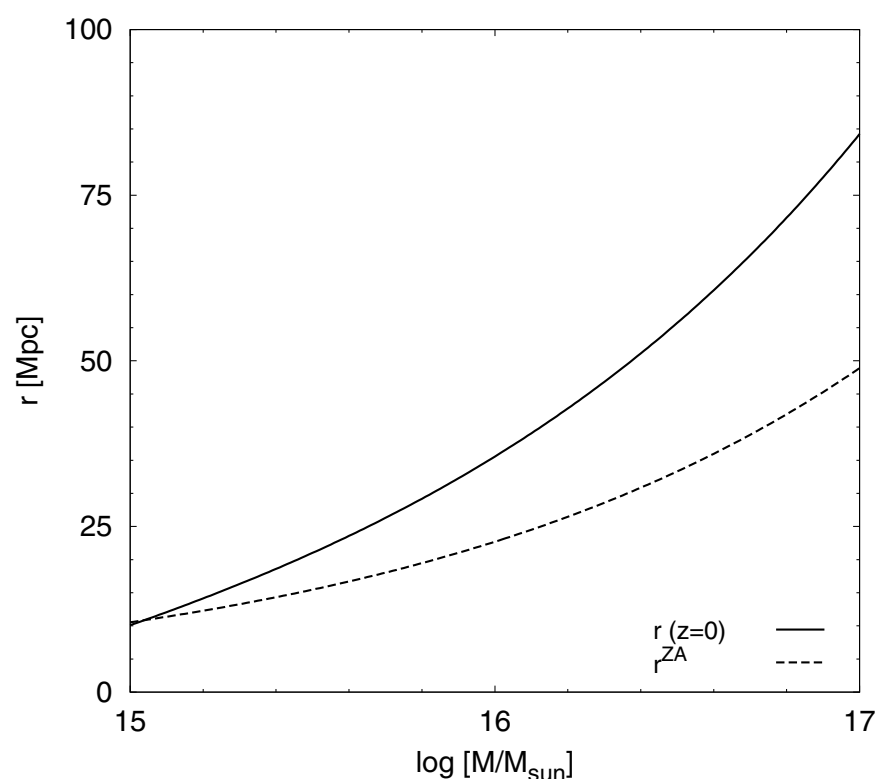

Fig. 6. Radii at present, $r(z=0)$, and zero proper acceleration radii, $r^{\mathrm{ZA}}$, of shells enclosing masses $M$.

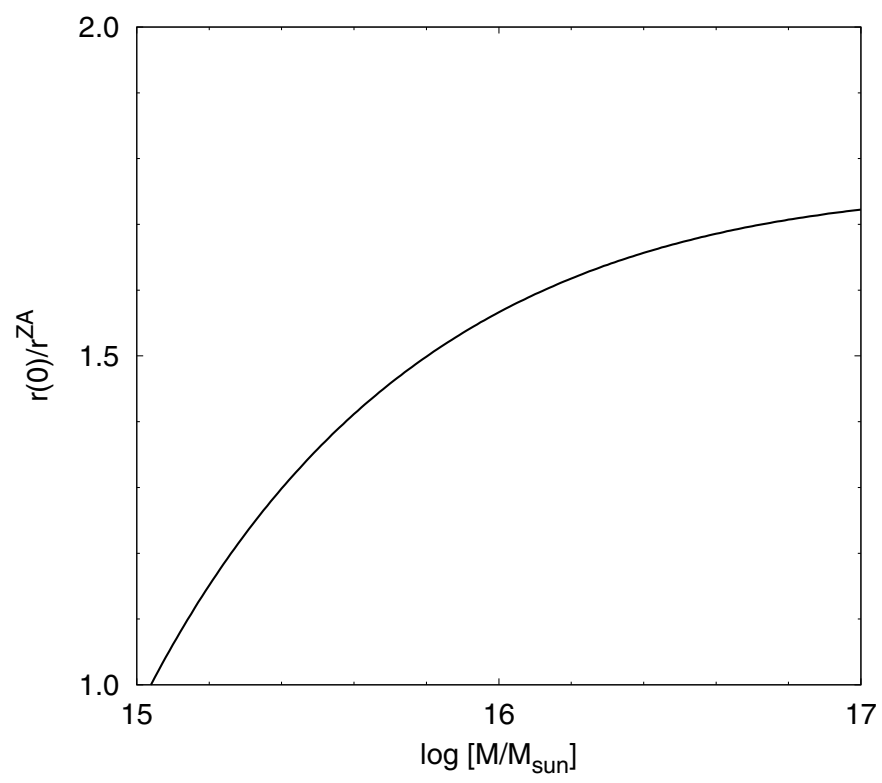

Fig. 7. $r(0) / r^{\mathrm{TA}}$ for shells enclosing masses $M$ at present.

At $z=0$, shells with radii, $r_{i}(z=0)$, greater than $\left[r^{\mathrm{ZA}}\right]_{0}=$ $10.87 \mathrm{Mpc}$ have crossed their zero proper acceleration radii, $r_{i}^{\mathrm{ZA}}$ (see Fig. 2), and show positive proper accelerations. These shells enclose masses, $\mathcal{M}_{i}$, greater than $\left[\mathcal{M}^{\mathrm{ZA}}\right]_{0}=1.10 \times 10^{15} \mathcal{M}_{\odot}$. Further shells are going to enclose larger masses (see Fig. 1). Therefore, in Eq. (106), although the matter gravitational term is diminished by the radii of farther shells, it is enlarged because such shells also show larger masses.

From Eq. (106), the matter gravitational term of a shell $i$ is neglected with respect to its cosmological constant term at redshift $z$ which fulfills

$r_{i}(z) \gg 2^{1 / 3}\left(\frac{3 c^{2}}{8 \pi} \frac{\mathcal{M}_{i}}{\bar{\rho}_{\Lambda}}\right)^{1 / 3}=2^{1 / 3} r_{i}^{\mathrm{ZA}}$.

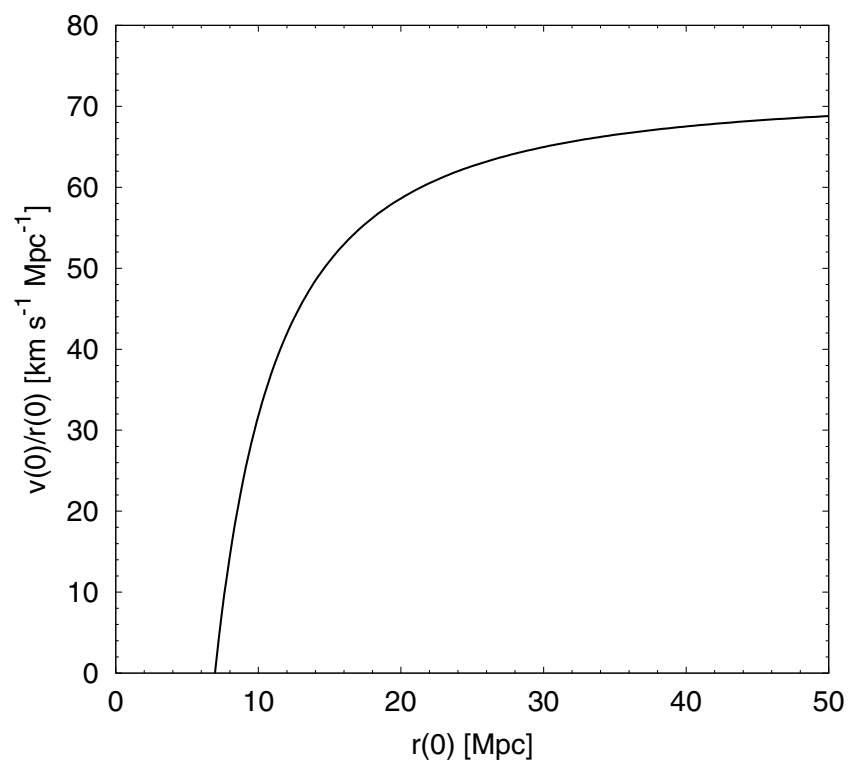

Fig. 8. $v(z=0) / r(z=0)$ for shells as a function of their radii at present.

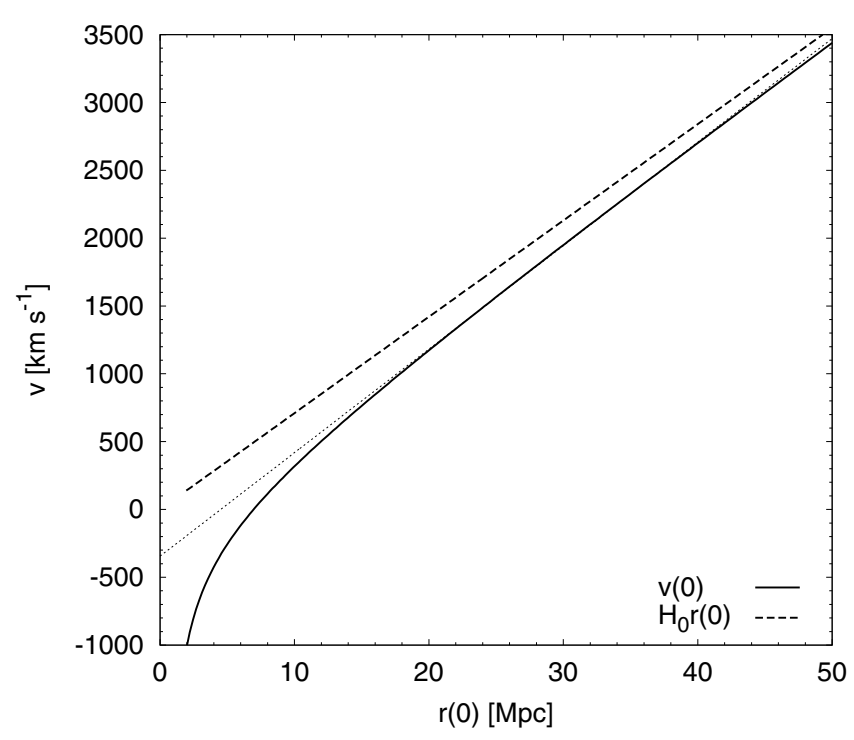

Fig. 9. Proper velocity of shells at $z=0, v(0)$, and the current velocity due to the Hubble flux, $H_{0} r(0)$, as a function of their radii, $r(0)$. The dotted line is the best-fitting straight line to $v(0)$ between $20 \mathrm{Mpc}$ and $40 \mathrm{Mpc}$ (see the text).

Figure 6 shows proper radii at present, $r(z=0)$, and zero proper acceleration radii, $r^{\mathrm{ZA}}$, of shells that enclose different masses, $\mathcal{M}$. In Fig. 7, $\left(r(0) / r^{\mathrm{TA}}\right)$ is shown for shells enclosing different masses $\mathcal{M}$. The curve in Fig. 7 indicates that the relation (110) is not fulfilled at present, and therefore, the asymptotic behaviour given by Eq. (109) has not been reached by any shell.

The radius at $z=0$ of a shell $i$ fulfilling $\mathcal{M}_{i} \gg\left[\mathcal{M}^{\mathrm{ZA}}\right]_{0}$ shows the asymptotic behavior

$r_{i}(0) \rightarrow 1.755 r_{i}^{\mathrm{ZA}}$.

According to Fig. 2, $z_{i}^{\mathrm{TA}} \rightarrow z_{U}^{\mathrm{TA}}=0.755$; therefore, Eq. (111) tell us that shells enclosing masses $\mathcal{M}_{i} \gg\left[\mathcal{M}^{\mathrm{ZA}}\right]_{0}$ tend to expand with the universe at present, as was expected. 
Figure 8, shows quotients between proper velocities of shells and their radii at present, $v(0) / r(0)$, as a function of their present radii, $r(0)$. It can be seen that these quotients are not constant. In our average cluster model, this could mean that the Hubble-Sandage paradox could not be present. In Fig. 9, proper velocities of shells at present, $v(0)$, are shown as a function of their radii, $r(0)$; in the same figure velocities due to the Hubble flux, $H_{0} r(0)$, are also represented. In this figure, proper velocities beyond the zero proper acceleration radius at present, $\left[r^{\mathrm{ZA}}\right]_{0}=10.87 \mathrm{Mpc}$, can be compared with velocities due to the Hubble flux. From about $20 \mathrm{Mpc}$ up to $40 \mathrm{Mpc}$, in Fig. 9, we can see that the relation between $v(0)$ and $r(0)$ is a straight line, which does not cross the origin $(v(0)=0, r(0)=0)$. Thus, while velocities due to the Hubble flux behave as $H_{0} r$, proper velocities from 20 up to $40 \mathrm{Mpc}$ fulfill $H_{C} r+C$ with $H_{C}$ and $C$ being constants (the best-fitting straight line to $v(0)$ is achieved for $H_{C}=76.30 \mathrm{~km} \mathrm{~s}^{-1} \mathrm{Mpc}^{-1}$ and $C=-344.2 \mathrm{~km} \mathrm{~s}^{-1}$ ). Finally, we should add that a similar behavior has been observed in models where the filtering scale $R_{\mathrm{f}}$ is taken of the order of galactic scales or of galaxy group scales. So, the same conclusion with respect to the Hubble-Sandage paradox is obtained for these smaller systems.

\section{Conclusions}

1. The goal of this work has been to investigate the infall region of CDM clusters, which are modeled by the attractive gravitational strength generated by matter and by the repulsive effect exerted by the cosmological constant.

2. For this purpose, we have dealt with a spherical infall model. Thus, the non-linear collapse of non-relativistic spherical mass shells has been studied for an average cluster, where a background of non-relativistic matter, radiation, and the cosmological constant have been included.

3. In this study, we have found that each cluster has a maximum mass which can virialize. In the case of the average cluster, this mass is $\left[\mathcal{M}^{\mathrm{VIR}}\right]_{\mathrm{MAX}}=8.13 \times 10^{14} \mathcal{M}_{\odot}$. In the evolution of shells enclosing masses $\mathcal{M}>\left[\mathcal{M}^{\mathrm{VIR}}\right]_{\mathrm{MAX}}$, the shells reach zero proper acceleration at redshifts at which the attractive and repulsive effects are balanced. At smaller redshifts, their proper accelerations are positive, so they will never collapse.

4. Shells enclosing masses $\mathcal{M} \gg\left[\mathcal{M}^{\mathrm{VIR}}\right]_{\text {MAX }}$ reach zero proper acceleration at redshifts which asymptotically tend to 0.755 . This asymptotic value coincides with the redshift at which the repulsive effect of the cosmological constant equals the gravitational strength due to the background of nonrelativistic matter $(\ddot{a}=0)$.

5. Some results obtained for our average cluster at $z=0$ can be summarized as follows:

a) There is a shell that is virializing at present. It has a radius $\left[r^{\mathrm{VIR}}\right]_{0}=1.50 \mathrm{Mpc}$ and encloses a mass $\left[\mathcal{M}^{\mathrm{VIR}}\right]_{0}=$ $3.47 \times 10^{14} \mathcal{M}_{\odot}$; these values represent the mass and radius of the virialized core.

b) There is another shell whose proper velocity is equal to zero. This shell presents $\left[r^{\mathrm{TA}}\right]_{0}=6.85 \mathrm{Mpc}$ and $\left[\mathcal{M}^{\mathrm{TA}}\right]_{0}=6.76 \times 10^{14} \mathcal{M}_{\odot}$. Thus, we have found that $\left[\mathcal{M}^{\mathrm{TA}}\right]_{0}=1.95\left[\mathcal{M}^{\mathrm{VIR}}\right]_{0}$ (it differs appreciably from the case $\Lambda=0$ in a flat universe: $\left[\mathcal{M}^{\mathrm{TA}}(\Lambda=0)\right]_{0}=$ $\left.5.26\left[\mathcal{M}^{\mathrm{VIR}}(\Lambda=0)\right]_{0}\right)$; this result agrees with that derived by Rines \& Diaferio (2006) averaging over all clusters of the Fourth Data Release of the SDDS. The masses and radii of the virialized core and of the zero proper velocity shell of our average cluster at present are also consistent with results by Rines et al. (2003) for clusters studied in the CAIRNS project. It should be noted that the infall region between these two shells shows negative proper velocities.

c) A third shell with $\left[r^{\mathrm{ZA}}\right]_{0}=10.87 \mathrm{Mpc}$ and $\left[\mathcal{M}^{\mathrm{ZA}}\right]_{0}=$ $1.10 \times 10^{15} \mathcal{M}_{\odot}$ shows null proper acceleration. Let us again remember that the infall region between the zero proper velocity shell and the zero proper acceleration shell shows positive proper velocity and negative proper acceleration. Nevertheless only shells enclosing masses $6.76 \times 10^{14} \mathcal{M}_{\odot}<\mathcal{M}<8.13 \times 10^{14} \mathcal{M}_{\odot}$ in this region are able to virialize in the future. Shells enclosing masses $\mathcal{M}>8.13 \times 10^{14} \mathcal{M}_{\odot}$ reach a positive proper acceleration. Currently, regions outside the zero proper acceleration shell have positive acceleration.

6. Finally, at present $(z=0)$ and from approximately $20 \mathrm{Mpc}$ up to $40 \mathrm{Mpc}$ (i.e., beyond the zero proper acceleration shell), we must state that the relation between shell proper velocities and their radii could be ajusted by a straight line. This straight line differs from that of velocities due to the Hubble flux, as shown in Fig. 9.

\section{References}

Abell, G. O. 1958, ApJS, 3, 211

Bahcall, N. A. 1988, ARA\&A, 26, 631

Bardeen, J. M., Bond, J. R., Raise, N., \& Szalay, A. S. 1986, ApJ, 304, 15 (BBKS)

Bond, J. R. 1988, The Early Universe, eds. W. G. Unruh, \& G. W. Semenoff (Dordrecht: Reidel)

Caldwell, D. O. 1995, Nucl. Phys. B, 38, 394

Chaboyer, B., Denmarque, P., Kernan, P. L., \& Krauss, L. M. 1995, Science, 271, 957

Chernin, A. D. 2001, Physics-Uspekhi, 44, 1099

Chernin, A. D., Terikorpi, I. D., \& Baryshev, Yu. V. 2000, Adv. Space Res., 31, 459

Chernin, A. D., Karachentsev, I. D., Nasonova, O. G., et al. 2010, A\&A, 520, A104

Davis, M., \& Huchra, J. 1982, ApJ, 254, 437

Dolgachev, V. P., Domozhilova, L. M., \& Chernin, A. D. 2003, Astr. Rep., 47, 728

Dolgachev, V. P., Domozhilova, L. M., \& Chernin, A. D. 2004, Astr. Rep., 48, 787

Efstathiou, G., Bond, J. R., \& White, S. D. M. 1992, MNRAS, 258, 1

Efstathiou, G., Lasenby, S. L. B. A. N., Hobson, M. P., et al. 1998 [arXiv:astro-ph/9812226]

Ekholm, T., Lanoix, P., Paturel, G., Teerikorpi, P., \& Fouqué, P. 1999, A\&A, 351, 827

Ekholm, T., Lanoix, P., Teerikorpi, P., Fouqué, P., \& Paturel, G. 2000, A\&A, 355, 835

Fouqué, P., Solanes, J. M., Sanchis, T., \& Balkowski, C. 2001, A\&A, 375, 770

Gladders, M. D., \& Yee, H. K. C. 2005, ApJS, 157, 1

Goto, T., Hanami, H., Myungshin, I., et al. 2008, PASJ, 60, S531

Gunn, J. E., \& Gott, J. R. 1972, ApJ, 176, 1

Hao, J., McKay, T. A., Koester, B. P., et al. 2010, ApJS, 191, 254

Hoffman, G. L., \& Salpeter, E. E. 1982, ApJ, 263, 485

Huchra, J. P. 1988, in The Extragalactic Distance Scale, ASP, 257

Karachentsev, I. D. 2005, AJ, 129, 178

Karachentsev, I. D., \& Kashibadze, O. G. 2006, ApJ, 49, 3

Karachentsev, I. D., \& Novasona, O. G. 2010, MNRAS, 405, 1075

Karachentsev, I. D., Sharina, M. E., Makarov, D. I., et al. 2002, A\&A, 389, 812

Karachentsev, I. D., Dolphin, A. E., Tully, R. B., et al. 2006, AJ, 131, 1361

Karachentsev, I. D., Kashibadze, O. G., Makarov, D. I., \& Tully, R. B. 2009, MNRAS, 393, 1265

Lahav, O., Lilje, P. B., Primack, J. R., \& Rees, M. J. 1991, MNRAS, 251, 128

Lilje, P. B., \& Lahav, O. 1991, ApJ, 374, 29

Lynden-Bell, D. 1981, The Observatory, 101, 111

Membrado, M., \& Aguerri, J. A. L. 2004, A\&A, 424, 415

Membrado, M., \& Pacheco, A. F. 2012, Europhys. Lett., 100, 39004

Membrado, M., \& Pacheco, A. F. 2013, A\&A, 551, A68 
M. Membrado and A. F. Pacheco: Effects of the cosmological constant on cold dark matter clusters

Padmanabhan, T. 1995, Structure Formation in the Universe (Cambridge: Cambridge University Press)

Peacock, S. 1999, Cosmological Physics (Cambridge: Cambridge University Press)

Peebles, P. J. E. 1976, ApJ, 205, 318

Peebles, P. J. E. 1980, The large-Scale Structure of the Universe (Princeton: Princeton Univ. Press)

Peebles, P. J. E. 1984, ApJ, 284, 439

Perlmutter, S., Aldering, G., \& Deustua, S. 1998, Bull. Am. Astron. Soc., 29, 1351

Perlmutter, S., Aldering, G., Goldhaber, G., et al. 1999, ApJ, 517, 565

Raychaudhuri, A. K., Benerji, S., \& Banerjee, A. 1992, General Relativisty, Astrophysics and Cosmology (New York: Springer-Verlag)

Rines, K., \& Diaferio, A. 2006, AJ, 132, 1275

Rines, K, Geller M. J., Kurtz, M. J., \& Diaferio, A. 2003, AJ, 126, 2152

Sandage, A. 1986, ApJ, 307, 1
Sandage, A. 1999, ApJ, 527, 479

Schmidt, B., Sunzeff, N. B., Phillips, M. M., et al. 1998, ApJ, 507, 46

Silk, J. 1974, ApJ, 193, 525

Silk, J. 1977, A\&A, 59, 53

Spergel, D. N., Verde, L., Peris, H., et al. 2003, ApJS, 148, 175

Strukov, I. A., Skulachev, D. P., \& Klypin, A. A. 1987, Large Scale of the Universe, eds. J. Audouze, M.-C. Pelletar, \& H. Szalay (Kluwer, Dordrecht) IAU Symp., 130, 93

Sugiyama, N. 1995, ApJS, 100, 281

Teerikorpi, P., Bottinelli, L., Gouguenheim, L., \& Paturel, G. 1992, A\&A, 260, 17

Trimble, V. 1987, ARA\&A, 25, 425

Weinberg, S. 1987, Phys. Rev. Lett., 59, 2607

Wen, Z. L., \& Han, J. L. 2011, ApJ, 734, 68

Wen, Z. L., Han, J. L., \& Liu, F. S. 2009, ApJS, 183, 197

Yahil, A., Sandage, A., \& Tammann, G. A. 1980, ApJ, 242, 448 